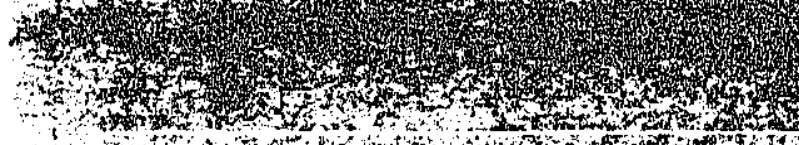

-

wat

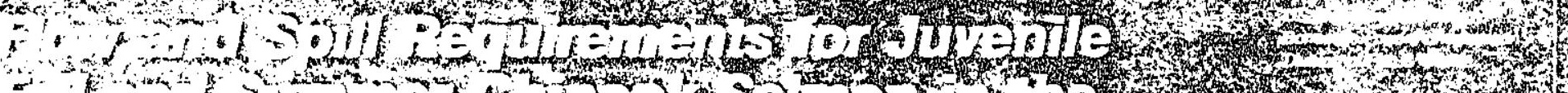

a -

ond

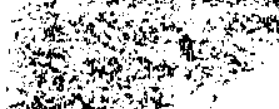

witis

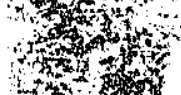

$+2$

Fin

th

ond

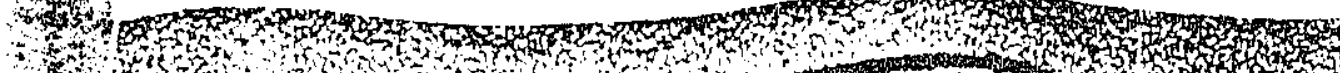

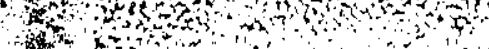

$4 x$

(t)

3

to

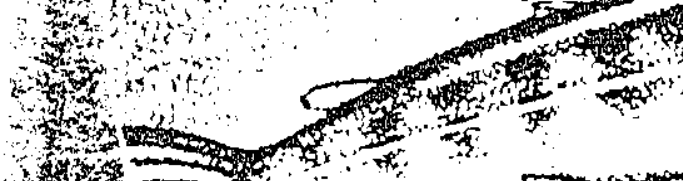

s

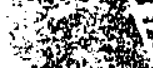

.

更

ঙे
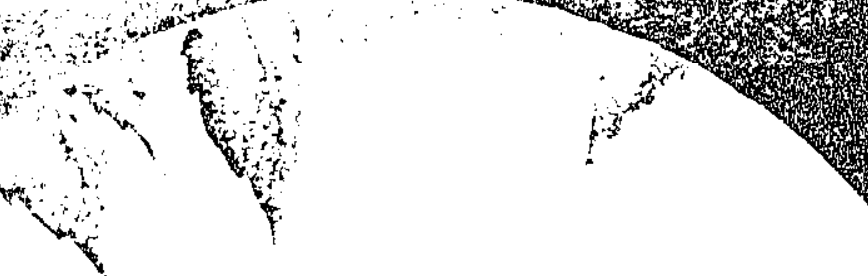
This report was funded by the Bonneville Power Administration (BPA), U.S. Department of Energy, as part of BPA's program to protect, mitigate, and enhance fish and wildlife affected by the development and operation of hydroelectric facilities on the Columbia River and its tributaries. The views in this report are the author's and do not necessarily represent the views of $\mathrm{BPA}$.

For copies of this report, write:

Bonneville Power Administration

Divi si on of Fish and Wildlife

Public Information Officer - PJ

P.0. Box 3621

Portland, OR 97208 


\title{
EFFECTS OF FLOW ON THE MIGRATORY BEHAVIOR AND SURVIVAL \\ OF JUVENILE FALL AND SUMMER CHINOOK SALMON \\ IN JOHN DAY RESERVOIR
}

by

David R. Miller

and

Carl W. Sims

Annual Report of Research (FY83)

Financed by

Bonneville Power Administration

(ContractDE-A179-83BP39645)

\author{
and \\ Coastal Zone and Estuarine. Studies Division \\ Northwest and Alaska Fisheries Center \\ National Marine Fisheries Service \\ National Oceanic and Atmospheric Administration \\ 2725 Montlake Boulevard East \\ Seattle, Washington 98112
}

June 1984 
ABS TRACI

The National Marine Fisheries Service in cooperation with the Bonneville Power Administration is conducting a 6-year study of the effects of instream river flow on the passage time, survival, and migrational behavior of juvenile fall and summer (O-age) chinook salmon in John Day Reservoir. In 1983, the final year of juvenile sampling in the reservoir, research activities continued to refine flow/travel time relationships and distributions1 behavior of 0-age chinook salmon.

Fifteen groups $(72,559$ fish) of marked O-age chinook salmon were wire-tagged, branded, and released into the $t$ ailrace at McNary Dam, and thirty-two groups $(22,206 \mathrm{fish})$ were branded and released into the reservoir at various other sites.

Sampling at John Day Dam, utilizing the a irlift pump.system in the B and C slots of Turbine Intake Unit 3, captured 82.698 subyearling chinook salmon including 640 mark recoveries. Additional marks (458) were recovered from purse seine samples taken at various sites throughout the reservoir.

Weekly mean fork lengths of O-age chinook salmon captured at McNary and John Day Dams and in the reservoir by purse seine ranged from $103 \mathrm{~mm}$ in mid-June to $166 \mathrm{~mm}$ in mid-December. Fish captured at the John Day Dam monitoring facility and hy purse seine throughout the reservoir were in excellent condition. Preliminary analysis of stomach samples taken in 1982 and 1983 from purse seine catches indicates active feeding is taking place in the reservoir. 
The average passage time of the fastest moving marked 0 -age chinook salmon from McNary Dam to John Day Dam was 11 days (based on 25th percentile of mark recaptures). The average reservoir residence time (calculated from the median date of the mark recoveries from each group at John Day Dam) was 22 days.

Regression analysis was used to develop a description of the relationship of river flow to the rate of downstream movement of 0 -age chinook salmon in John Day Reservoir in 1983. The slope of this line and the correlation coefficient (R) were not significantly different from zero. 


\section{INTRDDUCTION}

The National Marine Fisheries Service (NMFS), in cooperation with the Bonneville Power Administration (BPA), is conducting a 6-year study of the effects of flow on the migratory behavior and survival of juvenile fall and summer chinook salmon in John Day Reservoir (Lake Umatilla.

The objectives of the study are to:

1. Define the effect of instream flow on the passage time and rata of downstream movement of O-age chinook salmon in John Day Reservoir.

2. Define the effect of instream flow levels on the distribution and passage behavior of O-age chinook salmon in John Day Reservoir.

3. Define the effect of reservoir passage time on relative survival of 0 -age chinook salmon.

The study is divided into 3 years $(1981-83)$ of juvenile marking and recovery of marks to define flow/travel time relationships and 3 years (1984-86) of recoveries of marked adults. to define flow/survival relationships. This report summarizes results of research activities conducted during the final year of juvenile marking and recovery.

METHODS

Groups of 0-age chinook salmon from early (16 June - 8 July), middle (13 - 29 July), and late (5 August - 2 september) segments of the 1983 migration entering John Day Reservoir ware collecte d at McNary Dam, wire-tagged, freeze branded, and released into the tailrace below the dam. Recoveries of these marks from the airlift fish collection facility at John Day Dam (Sims et al. 1981) were used to define reservoir travel and, residence time. 
Table 1.--Purse seine sampling transect locations by river mile and kilometer in John Day Reservoir, 1983.

\begin{tabular}{ccl}
\hline River Mile $(\mathrm{RM})$ & River Kilometer $(\mathrm{RKm})$ & \multicolumn{1}{c}{ Area } \\
\hline 216 & 348 & John Day Dam Forebay \\
222 & 357 & Goodnoe \\
232 & 373 & Blalock \\
242 & 389 & Arlington \\
253 & 407 & Willow Creek \\
267 & 430 & Crow Butte \\
\hline
\end{tabular}


Table Z.--Summary of 0-age chinook salmon wire-tagged, cold branded, and released in the McNary Dam tailrace and recovered at John Day Dam, 1983.

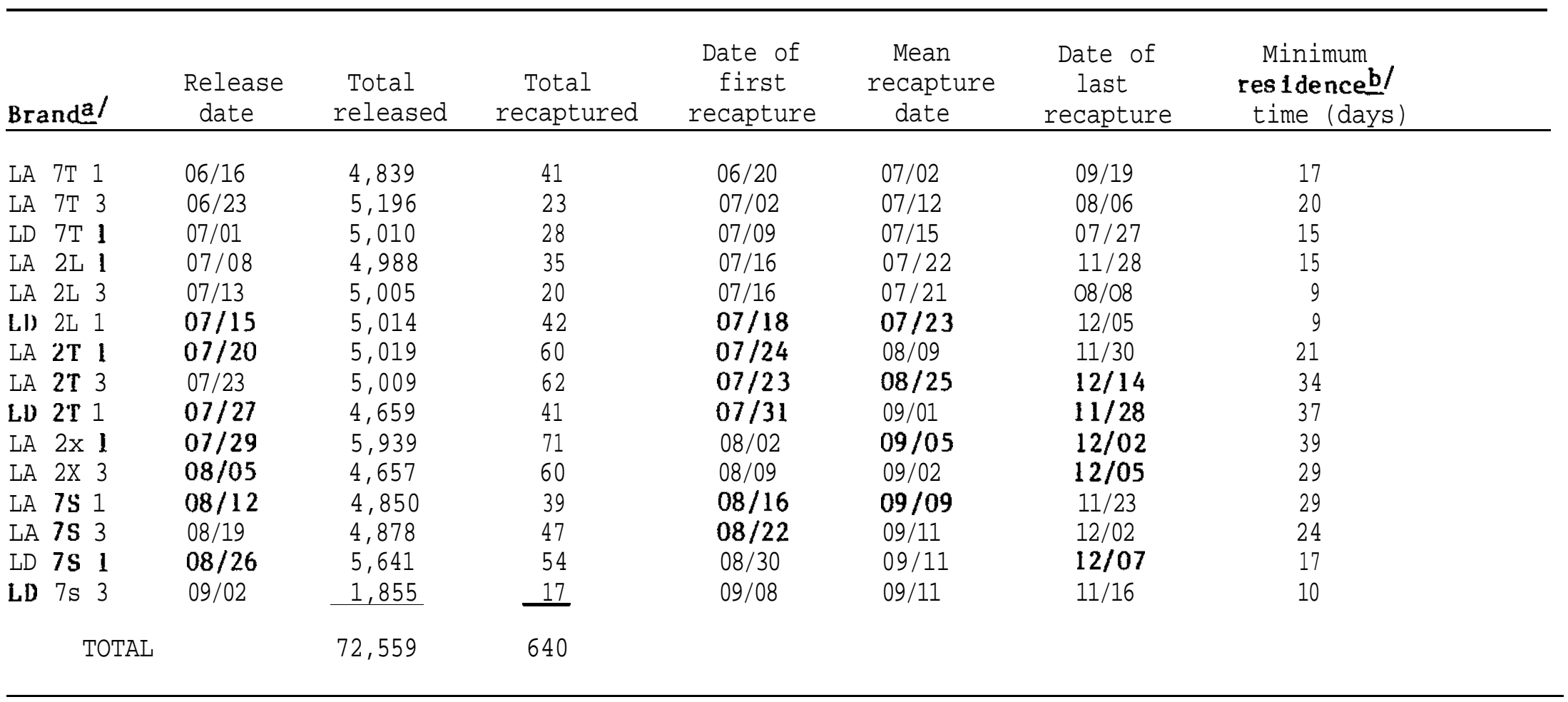

af Position, brand, and orientation. LA indicates left anterior, LD indicates left dorsal. Orientation refers to rotation of the brand around its center point, (i.e., 1 equals normal orientation ID, 2 equals $\boldsymbol{B}, 3$ equals $\mathbf{q 1}$, and 4 equals $\boldsymbol{A}$ ).

b/ Difference between median date of recovery and release date. 
salmon distribution and migrational behavior in John Day Reservoir.

Purse seine catches were processed aboard the Columbia. Catches at John Day Dam were processed on site. All fish were anesthetized with MS-222, counted, and examined for marks. Those fish to be marked were freeze branded. A subsample was measured for fork-length. After processing, all fish were allowed to recover from the anesthetic and released on site.

\section{RESULTS AND DISCUSSION}

A total of 94,765 0-age chinook salmon were marked and released into John Day Reservoir in 1983. Fifteen groups (72,559 fish) were wire-tagged, branded, and released into the tailrace at McNary Dam (Table 2). of the fifteen groups released, four groups $(20,033)$ were released during the early migration (29 June - 8 July), six groups $(30,645)$ during the middle migration (13 - 29 July), and five groups $(21,881)$ during the late migration (5 August - 2 september). An additional 22,206 fish from purse seine catches were marked and released at the six reservoir sampling transects (Table 3).

Total passage of 0 -age chinook salmon at John Day Dam was estimated at 7.5 million fish. The airlift collection facility at John Day Dam captured 82,698 0-age chinook salmon between 27 May and 16 December 1983 (Table 4). Airlift catches included 640 marked fish from the McNary tailrace releases and 411 marked fish from purse seine releases. Detailed mark recovery information is included in Appendix Table A-1.

Purse seine sampling began on 29 June and was maintained on a 3-day/week schedule, when possible, through 29 September. One hundred 
Table 3.--Summary of O-age chinook salmon captured by purse seining, cold branded, and released at various locations in John Day Reservoir, 6 July - 29 september 1983.

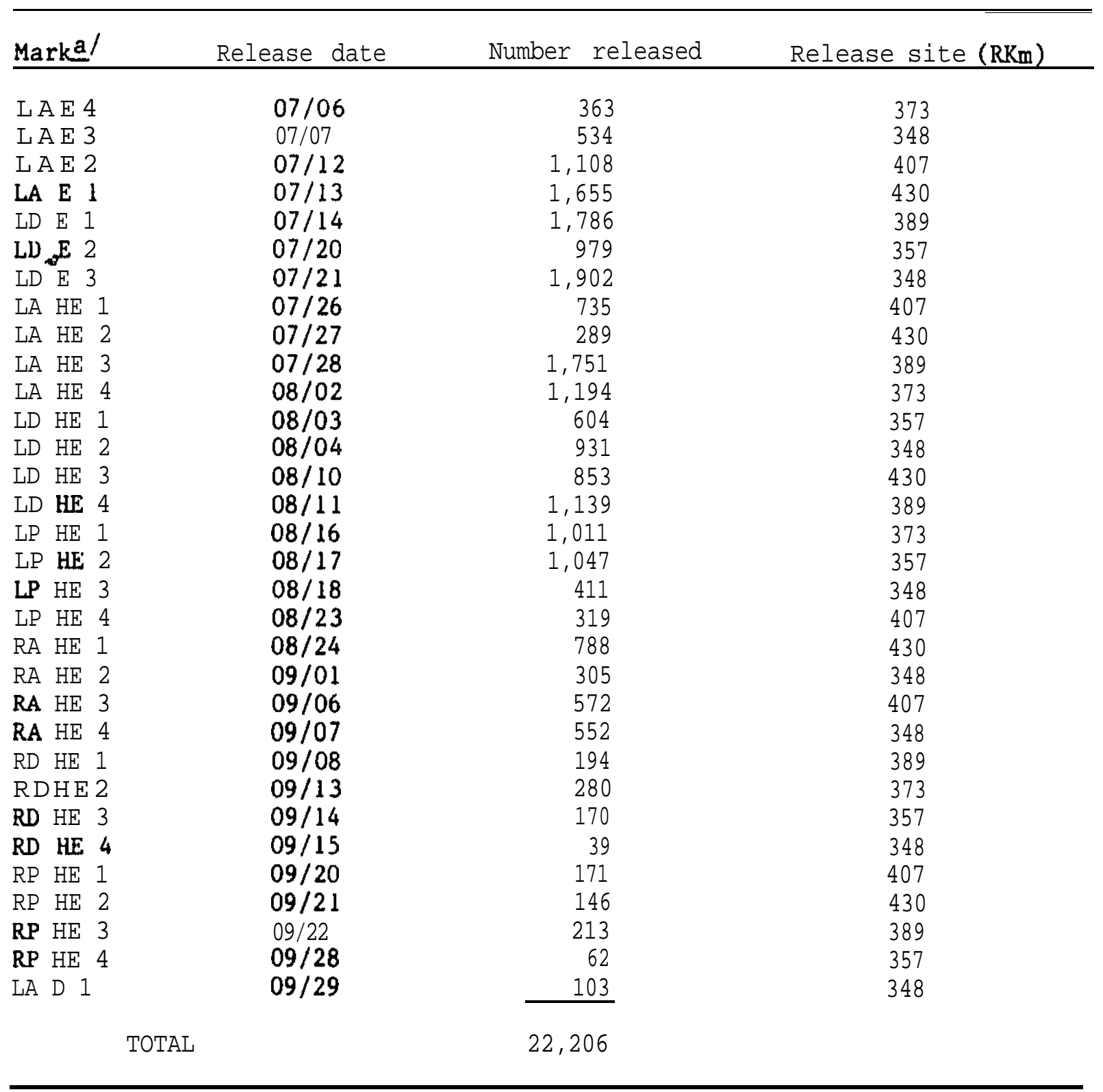

a/ Position, brand. and orientation. LA indicates left anterior, LD indicates left dorsal. Orientation refers to rotation of the brand around its center point, (i.e., 1 equals normal orientation ID, 2 equals $\boldsymbol{\xi}, 3$ equals $\boldsymbol{Q} \mathbf{I}$, and 4 equals $\boldsymbol{\theta}$ ). 
Table 4.--Weekly catch of subyearling chinook salmon from Turbine Intake Gatewells $3 \mathrm{~A}$ and $3 \mathrm{~B}$ at John Day Dam and weekly estimated passage by John Day Dam 27 May to 16 December 1983.

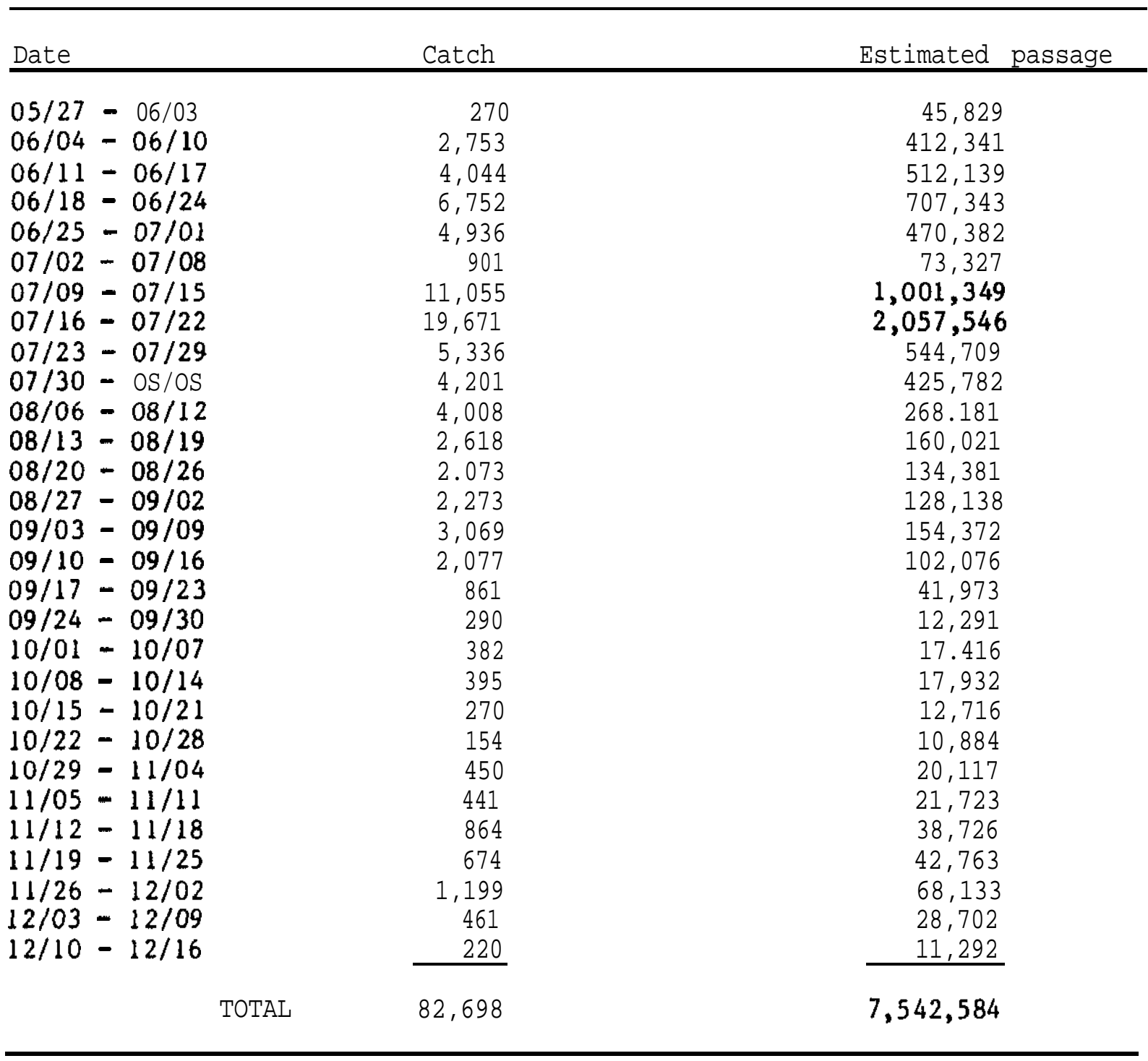


thirty-five (135) purse seine sets were made, and 22,484 0-age chinook salmon were captured (Tables 5 and 6 ). Purse seine catches included 345 marks from the McNary Dam tailrace releases and 113 marks from purse seine releases. Detailed purse seine mark recovery information is included in Appendix Table A-2.

Mean fork lengths of O-age chinook salmon captured at McNary and John Day Dams and at purse seine sites throughout John Day Reservoir are shown in Table 7. Mean fork length increased from $103 \mathrm{~mm}$ (mid-June) to $166 \mathrm{~mm}$ (mid-December). Gross observations of subyearling migrants as they were examined for marks at both John Day Dam and in reservoir purse seine catches showed the fish to be in excellent condition. Preliminary analysis of stomach samples taken at the purse seine sites in 1982 and 1983 demonstrated a low number of empty stomachs and a high percentage of stomachs that were > half full. These data and the growth rate indicated by the weekly mean fork lengths indicated that the fish were actively feeding in the reservoir.

Incidental purse seine catches of species other than juvenile salmonids in John Day Reservoir are summarized in Table 8. Juvenile shad were again the most abundant species taken, only small numbers of other species were caught. Numbers and distribution of squawfish were similar to the 1982 catch.

\section{Migrational Behavior}

The 1983 migration of O-age chinook salmon began to enter John Day Reservoir in early May and peaked at John Day Dam in mid-July. Fish. were still being taken at John Day Dam on 16 December when monitoring was discontinued. The average reservoir residence time, based on median date 
Table 5.--A summary of purse seine catches of subyearling chinook salmon in John Day Reservoir by major area, 26 June to 29 September 1983.

\begin{tabular}{lcccc}
\hline Date & Area & No. sets & Total catch & Catch/set \\
\hline June-July & Lower (RKm 348-378) & 24 & 4,056 & 193 \\
& Middle (RKm 385-431) & 17 & 7.324 & 431 \\
August & Lower & 31 & 5,198 & 168 \\
& Middle & 8 & $\mathbf{3 , 0 9 9}$ & 387 \\
September & Lower & 36 & 961 & $\mathbf{9 7}$ \\
& Middle & $\mathbf{1 9}$ & $\mathbf{1 , 8 4 6}$ & 167 \\
& TOTAL & 135 & 22,484 & \\
\hline
\end{tabular}


Table 6.--Weekly summary of purse seine catches in John Day Reservoir, 26 June to 29 September 1983.

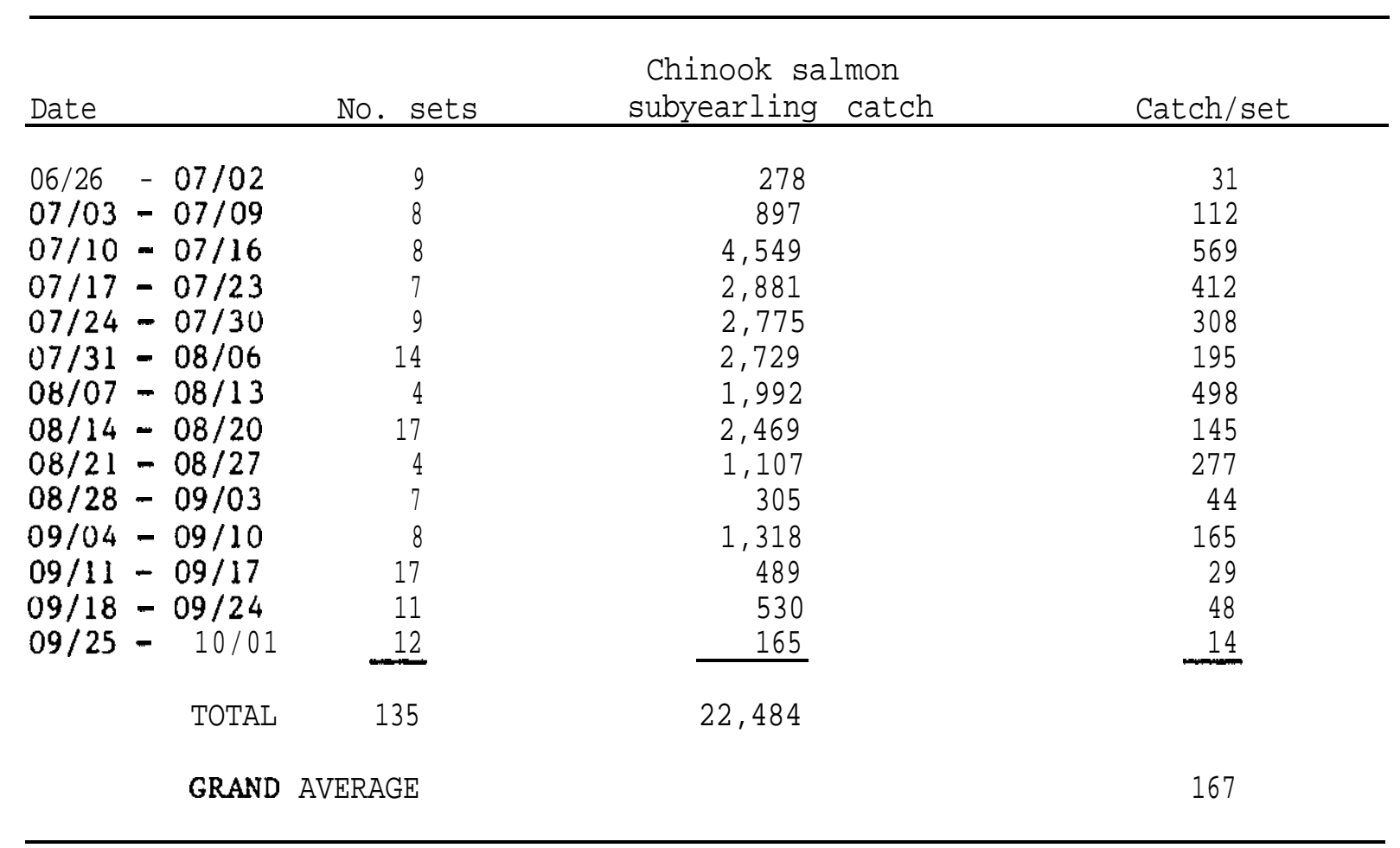


Table 7.--Weekly mean fork lengths in millimeters of 0-age chinook salmon captured at McNary and John Day Dam and by purse seine in John Day Reservoir, 18 June to 16 December 1983.

\begin{tabular}{|c|c|c|c|}
\hline \multirow[b]{2}{*}{ Date } & \multicolumn{3}{|c|}{ Fork length (mm) } \\
\hline & $\begin{array}{l}\text { McNary Dam } \\
\text { catch }\end{array}$ & $\begin{array}{c}\text { John Day Dam } \\
\text { catch }\end{array}$ & $\begin{array}{c}\text { Purse Seine } \\
\text { catch }\end{array}$ \\
\hline $\begin{array}{l}06 / 11-06 / 17 \\
06 / 18=06 / 24 \\
06 / 25-07 / 01 \\
07 / 02=07 / 08 \\
07 / 09-07 / 15 \\
07 / 16=07 / 22 \\
07 / 23-07 / 29 \\
07 / 30-08 / 05 \\
08 / 06-08 / 12 \\
08 / 13=08 / 19 \\
08 / 20-08 / 26 \\
08 / 27=09 / 02 \\
09 / 03-09 / 09 \\
09 / 10-09 / 16 \\
09 / 17-09 / 23 \\
09 / 24-09 / 30 \\
10 / 01-10 / 07 \\
10 / 08-10 / 14 \\
10 / 15-10 / 21 \\
10 / 22-10 / 28 \\
10 / 29-11 / 04 \\
11 / 05=11 / 11 \\
11 / 12-11 / 18 \\
11 / 19-11 / 25 \\
11 / 26-12 / 02 \\
12 / 03-12 / 09 \\
12 / 10-12 / 16\end{array}$ & $\begin{array}{r}99 \\
97 \\
93 \\
104 \\
116 \\
113 \\
121 \\
128 \\
129 \\
131 \\
135 \\
142\end{array}$ & $\begin{array}{r}109 \\
107 \\
98 \\
107 \\
115 \\
114 \\
116 \\
120 \\
128 \\
133 \\
134 \\
137 \\
141 \\
145 \\
147 \\
149 \\
149 \\
148 \\
153 \\
152 \\
153 \\
154 \\
157 \\
160 \\
159 \\
164 \\
166\end{array}$ & $\begin{array}{r}96 \\
103 \\
110 \\
115 \\
110 \\
120 \\
126 \\
129 \\
136 \\
135 \\
142 \\
147 \\
146 \\
148\end{array}$ \\
\hline
\end{tabular}


Table B.--Catch summary of salmonid and nonsalmonid fish captured by purse seine in John Day Reservoir, June to September 1983.

\begin{tabular}{|c|c|c|c|c|c|}
\hline \multirow[b]{2}{*}{ Species } & \multicolumn{5}{|c|}{ Number taken } \\
\hline & June & July & August & September & Total \\
\hline Subyearling chinook salmon & 278 & 11,102 & 8,297 & 2,807 & 22,484 \\
\hline Yearling chinook salmon & 10 & 11 & & & \\
\hline Jack chinook salmon & & & & 3 & 3 \\
\hline Adult chinook salmon & & & & 11 & 11 \\
\hline Juvenile sockeye salmon & 3 & 32 & 22 & 3 & 60 \\
\hline Juvenile steelhead & & 1 & 2 & 1 & 4 \\
\hline Adult steelhead & 1 & 2 & 11 & 38 & 52 \\
\hline Juvenile black bass & & 1 & & & 1 \\
\hline Juvenile whitefish & & 11 & 3 & & 14 \\
\hline Adult whitefish & & & 1 & & 1 \\
\hline Juvenile shad & & & 3,050 & 26,500 & 29,550 \\
\hline Adult shad & & 11 & 44 & & 55 \\
\hline Adult squawfish & & 17 & 62 & 11 & 90 \\
\hline Adult sucker & & 1 & & & I \\
\hline Peamouth chubs & & 4 & & & 4 \\
\hline Chiselmouth chubs & & 9 & 1 & & 10 \\
\hline
\end{tabular}


of mark recaptures, of branded O-age chinook salmon released into the McNary Dam tailrace in 1983, was 21 days (range 3-155+) (Table 9). As in 1981 and 1982, the average reservoir residence time in 1983 suggests that a large percentage of the O-age chinook salmon entering John Day Reservoir are not actively smolting.

Purse seine recoveries of marked subyearling chinook salmon released at various locations within the reservoir (excluding the McNary Dam tailrace releases) also indicate, as in 1981 and 1982, that a significant number of the fish are in a nonsmolting condition. Nearly $60 \%$ of the recoveries (66 of 111) were either at the same site (19) or upstream (47) from the original release site (Table 10). Such behavior is not representative of actively smolting fish.

\section{Flow/Travel Time Relationships}

Travel time (based on 25th percentile of mark recaptures) from McNary Dam to John Day Dam was calculated for the 15 groups of marked fish released into the McNary Dam tailrace in 1983 (Table 11). Average river flow for the 10-day period following each release ranged from 120 to 243 thousand feet ${ }^{3} /$ second $(\mathrm{kcfs})$. The average travel time ranged from 7 to 20 days. Considerable variance in travel time occurred regardless, of river flow. Overall, average travel time for the early (13 d), middle (14 d), and late (11 d) groups were nearly the same, although river flows declined from an average of $212 \mathrm{kcfs}$ for the early group to $146 \mathrm{kcfs}$ for the late group.

A regression line was constructed by plotting the travel time in hours for each release group against the appropriate river flow for the $\mathbf{1 5}$ groups 


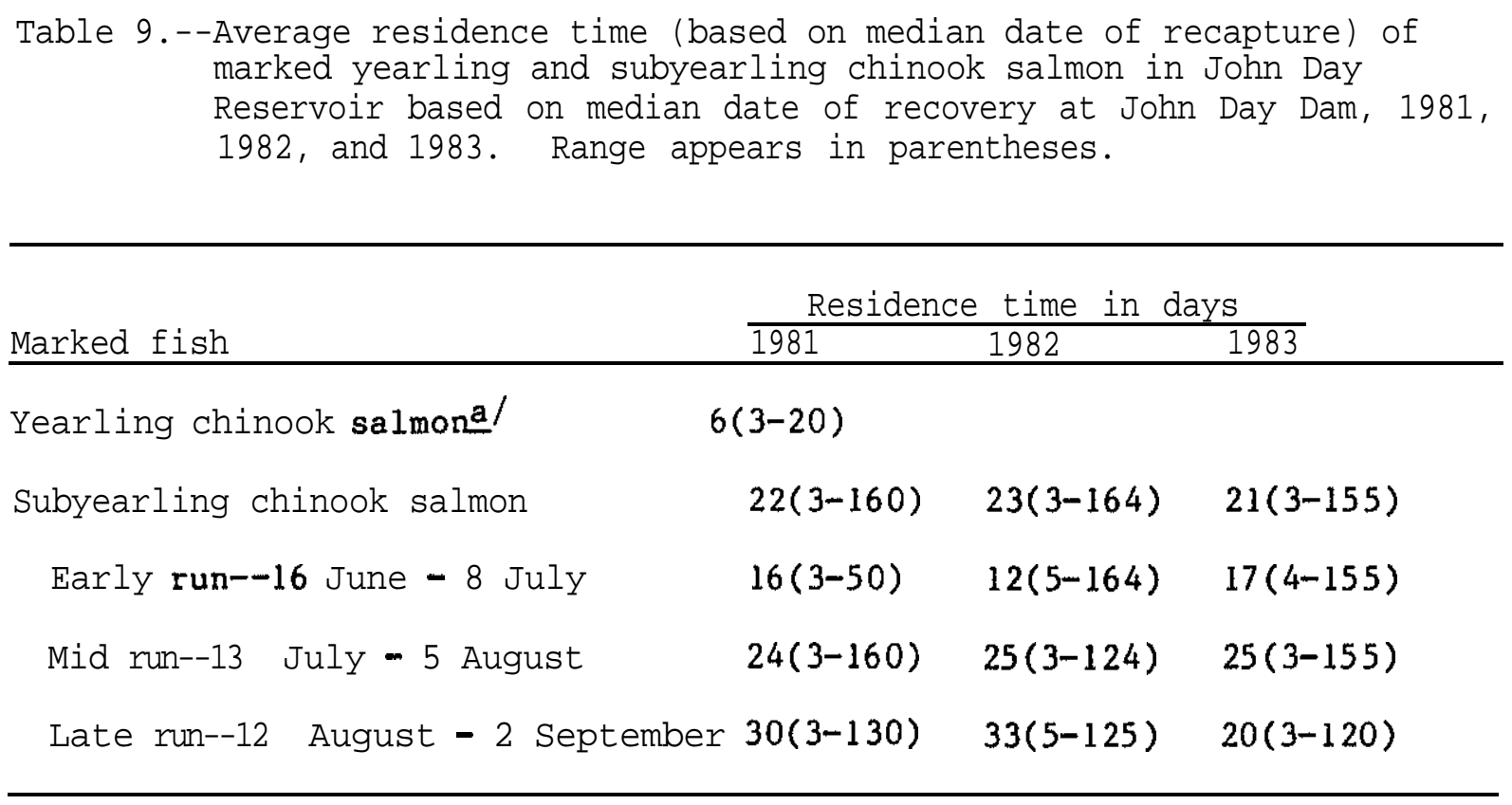

a/ Data from Sims et al. (1982). 
Table 10.--Purse seine recoveries of marked 0-age chinook salmon taken at or above their reservoir release site.

\begin{tabular}{|c|c|c|c|c|c|c|}
\hline $\begin{array}{l}\text { Release } \\
\text { site }\end{array}$ & $\begin{array}{l}\text { Recapture } \\
\text { site }\end{array}$ & $\begin{array}{l}\text { Date } \\
\text { released }\end{array}$ & $\begin{array}{l}\text { Date } \\
\text { recaptured }\end{array}$ & $\begin{array}{c}\text { Time interval } \\
\text { (days) }\end{array}$ & $\begin{array}{l}\text { Distance } \\
\text { traveled } \\
\text { upstream }\end{array}$ & $(\mathrm{Km})$ \\
\hline \multirow[t]{2}{*}{ RKm 373} & 389 & $07 / 06$ & $07 / 14$ & 9 & 16 & \\
\hline & 389 & $07 / 06$ & $07 / 14$ & 9 & 16 & \\
\hline \multirow[t]{4}{*}{348} & 430 & $07 / 07$ & $07 / 27$ & 21 & 82 & \\
\hline & 389 & & $07 / 28$ & 22 & 41 & \\
\hline & 373 & & $08 / 02$ & 27 & 25 & \\
\hline & 357 & & $08 / 17$ & 42 & 9 & \\
\hline 407 & 430 & $07 / 12$ & $08 / 24$ & 44 & 23 & \\
\hline \multirow[t]{4}{*}{389} & 430 & $07 / 14$ & $08 / 10$ & 28 & 41 & \\
\hline & 389 & & $08 / 11$ & 29 & 0 & \\
\hline & 389 & & $08 / 11$ & 29 & 0 & \\
\hline & 407 & & $09 / 06$ & 55 & 18 & \\
\hline \multirow[t]{2}{*}{357} & 407 & $07 / 20$ & $07 / 26$ & 7 & 50 & \\
\hline & 357 & & $08 / 03$ & 15 & 0 & \\
\hline \multirow[t]{16}{*}{348} & 407 & $07 / 21$ & $07 / 26$ & 6 & 59 & \\
\hline & 407 & & $07 / 26$ & 6 & 59 & \\
\hline & 389 & & $07 / 28$ & 8 & 41 & \\
\hline & 373 & & $08 / 02$ & 13 & 25 & \\
\hline & 357 & & $08 / 03$ & 14 & 9 & \\
\hline & 357 & & $08 / 03$ & 14 & 9 & \\
\hline & 357 & & $08 / 03$ & 14 & 9 & \\
\hline & 348 & & 08/04 & 15 & 0 & \\
\hline & 389 & & $08 / 11$ & 22 & 41 & \\
\hline & 373 & & $08 / 16$ & 27 & 25 & \\
\hline & 373 & & $08 / 16$ & 27 & 25 & \\
\hline & 430 & & $08 / 24$ & 35 & 82 & \\
\hline & 430 & & $08 / 24$ & 35 & 82 & \\
\hline & 373 & & $09 / 13$ & 55 & 25 & \\
\hline & 348 & & $09 / 15$ & 57 & 0 & \\
\hline & 430 & & $09 / 21$ & 63 & 82 & \\
\hline 407 & 430 & $07 / 26$ & $08 / 24$ & 30 & 23 & \\
\hline \multirow[t]{3}{*}{430} & 430 & $07 / 27$ & $08 / 10$ & 15 & 0 & \\
\hline & 430 & & $08 / 24$ & 29 & 0 & \\
\hline & 430 & & $08 / 24$ & 29 & 0 & \\
\hline \multirow[t]{5}{*}{389} & 389 & $07 / 28$ & $08 / 11$ & 15 & 0 & \\
\hline & 430 & & $08 / 24$ & 28 & 41 & \\
\hline & 407 & & $09 / 06$ & 41 & 18 & \\
\hline & 430 & & $09 / 07$ & 42 & 41 & \\
\hline & 389 & & $09 / 22$ & 57 & 0 & \\
\hline 373 & 373 & $08 / 02$ & $08 / 16$ & 15 & 0 & \\
\hline \multirow[t]{4}{*}{357} & 373 & $08 / 03$ & $08 / 16$ & 14 & 16 & \\
\hline & 373 & & $08 / 16$ & 14 & 16 & \\
\hline & 357 & & $08 / 17$ & 15 & 0 & \\
\hline & 357 & & $08 / 17$ & 15 & 0 & \\
\hline
\end{tabular}


Table I0.--cont.

\begin{tabular}{|c|c|c|c|c|c|}
\hline $\begin{array}{l}\text { Release } \\
\text { site }\end{array}$ & $\begin{array}{l}\text { Recapture } \\
\text { site }\end{array}$ & $\begin{array}{l}\text { Date } \\
\text { released }\end{array}$ & $\begin{array}{l}\text { Date } \\
\text { recaptured }\end{array}$ & $\begin{array}{c}\text { Time intervel } \\
\text { (days) }\end{array}$ & $\begin{array}{l}\text { Distance } \\
\text { traveled } \\
\text { upstream }(\mathbf{K m})\end{array}$ \\
\hline \multirow{10}{*}{348} & 407 & \multirow{10}{*}{$08 / 04$} & $09 / 20$ & 49 & 50 \\
\hline & 357 & & $09 / 28$ & 57 & 0 \\
\hline & 389 & & $08 / 11$ & 8 & 41 \\
\hline & 373 & & $08 / 16$ & 13 & 25 \\
\hline & 373 & & $08 / 16$ & 13 & 25 \\
\hline & 348 & & $08 / 18$ & 15 & 0 \\
\hline & 348 & & $08 / 18$ & 15 & 0 \\
\hline & 407 & & $09 / 06$ & 34 & 59 \\
\hline & 357 & & $09 / 14$ & 42 & 9 \\
\hline & 430 & & $09 / 21$ & 49 & 82 \\
\hline 430 & 430 & $08 / 10$ & $08 / 24$ & 15 & 0 \\
\hline \multirow[t]{2}{*}{373} & 407 & \multirow[t]{2}{*}{$08 / 16$} & $08 / 23$ & 8 & 34 \\
\hline & 389 & & $09 / 08$ & 24 & 16 \\
\hline \multirow[t]{2}{*}{357} & 357 & \multirow[t]{2}{*}{$08 / 17$} & $09 / 14$ & 29 & 0 \\
\hline & 407 & & $09 / 20$ & 35 & 50 \\
\hline \multirow[t]{4}{*}{348} & 407 & \multirow[t]{4}{*}{$08 / 18$} & $09 / 06$ & 20 & 59 \\
\hline & 407 & & $09 / 06$ & 20 & 59 \\
\hline & 430 & & $09 / 07$ & 21 & a2 \\
\hline & 389 & & $09 / 08$ & 22 & 41 \\
\hline \multirow[t]{2}{*}{407} & 407 & \multirow[t]{2}{*}{$08 / 23$} & $09 / 06$ & 15 & 0 \\
\hline & 430 & & $09 / 07$ & 16 & 23 \\
\hline \multirow[t]{2}{*}{348} & 373 & \multirow[t]{2}{*}{$09 / 01$} & $09 / 13$ & 13 & 25 \\
\hline & 389 & & $09 / 22$ & 22 & 41 \\
\hline
\end{tabular}


Table Il.--Recoveries of 0-age chinook salmon (wire-tagged, cold branded, and released in McNary Dam tailrace, 24 June to 3 september 1983) at John Day Dam.

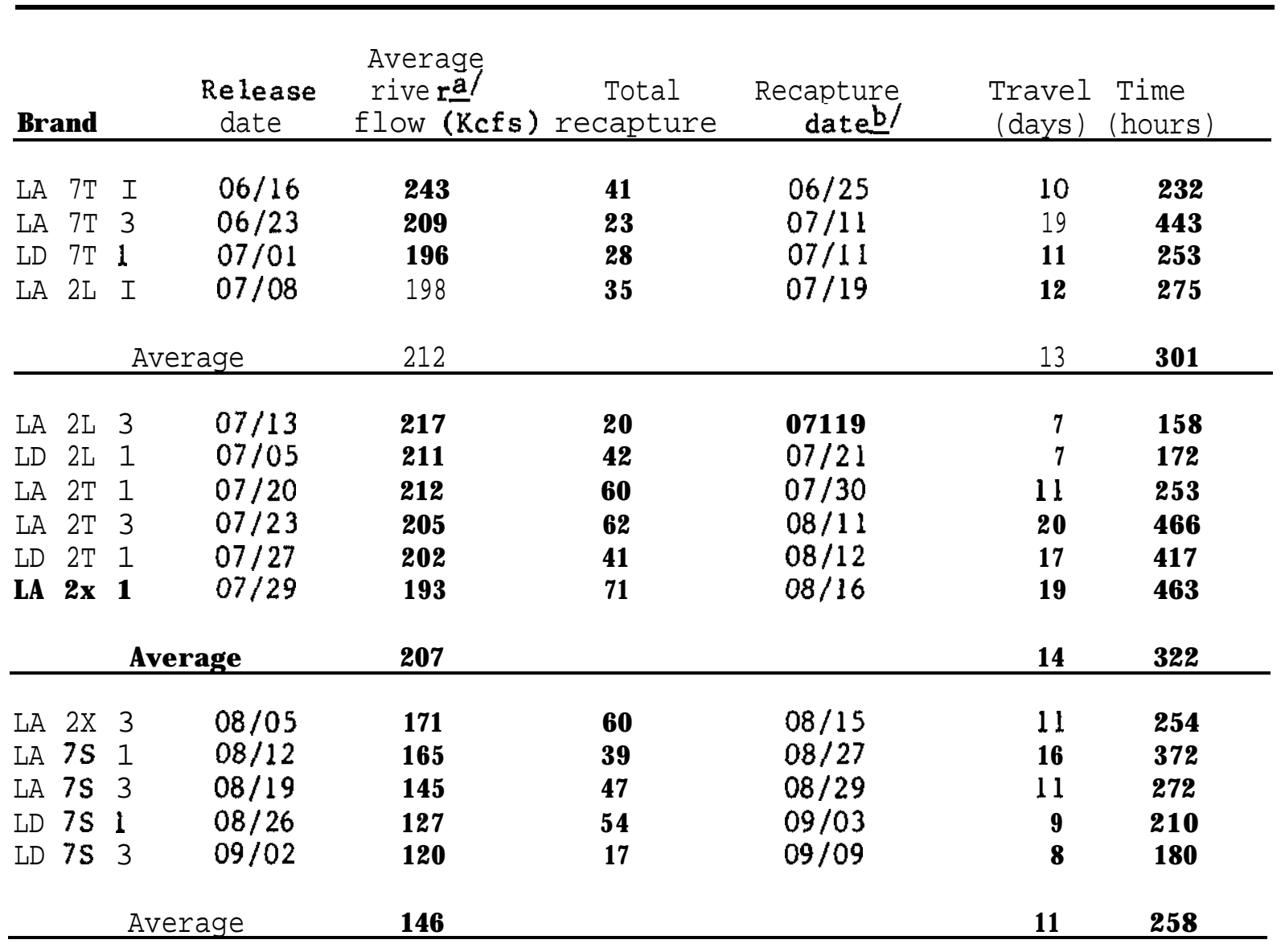

a/ For the IO-day period following each release date. Daily average river discharges at McNary Dam from 1 June to 30 September 1983 are given in Appendix A.

b/ 25 th percentile. 
in 1983 (Figure 1). The regression coefficient b (slope) of the line $\hat{\mathrm{y}}=95.8+0.53 \mathrm{x}$ was examined for significance by testing the null hypothesis that the population regression coefficient is equal to zero $\left(H_{0}: b=0\right)$. This was done by applying a sample t-test according to the formula: $\quad t=\frac{\mathrm{b}-\mathrm{B}}{\mathrm{s}_{\mathrm{b}}}$ where $\mathrm{b}=$ slope and $\mathrm{Sb}=$ standard error of the regression coefficient. The 15 data points developed showed a $t$ value of 1.02. For a two-sided test of significance with 13 degrees of freedom with $a=0.05$. the table of $t$ gave a value of \pm 1.771 . Therefore, $\mathrm{H}_{0}: \mathrm{b}=0$ " as accepted. Thus the slope (b) of this line did not possess a significant statistical difference from zero, and we found, as we did in 1981 and 1982, that there was no statistical evidence to indicate that river flows affected the rates of migration of 0 -age chinook salmon migrating through John Day Reservoir (Miller and Sims 1983).

\section{Flow/Survival Relationships}

Samples of the three segments of the O-age chinook salmon migration (early, middle, and late) entering John Day Reservoir in 1983 were wire-tagged and released into the McNary Dam tailrace. Adult returns from these releases will be used to determine relative survival of each segment. By plotting the survival estimates against the appropriate river flows, a regression line will be developed to determine if a significant flow/survival relationship existed.

\section{SUMMARY AND CONCLUSIONS}

1. Fifteen groups of O-age chinook salmon $(72,559$ fish) were wire-tagged and branded at McNary Dam and released into the McNary Dam 


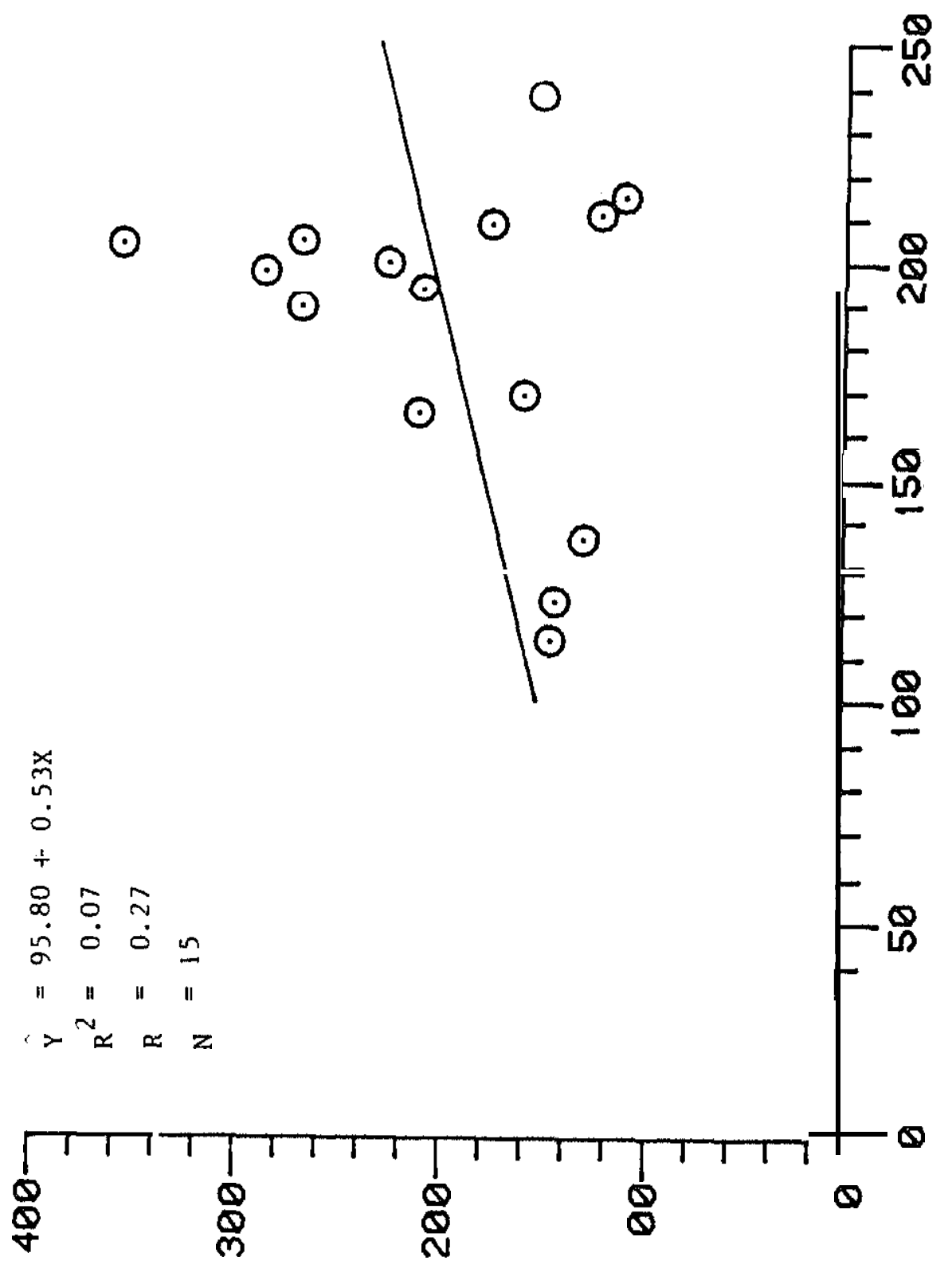

Figure 1.--Relationship of river flow to travel time of 0 -age chinook salmon through John Day Reservoir (:1cNary Dam tailrace to John Day Dam). 1983. 
tailrace during the period 16 June - 2 september 1983.

2. Additional mark releases of 22,206 purse seine captured 0-age chinook salmon were made at the six reservoir sampling transects.

3. The airlift collection facility in Turbine Unit 3, John Day Dam captured 82,698 0-age chinook salmon between 27 May and 16 December 1983. Total passage during this period, based on these collections, was estimated to be approximately 7.5 million fish.

4. Six hundred and forty marked O-age chinook salmon were recovered at John Day Dam.

5. Between 29 June and 29 September, 135 purse seine sets were made in John Day Reservoir. Purse seine catches totaled 22,484 0-age chinook salmon with 458 mark recoveries.

6. Mean fork lengths of O-age chinook salmon captured at McNary and John Day Dams and in the John Day Reservoir increased from $103 \mathrm{~mm}$ in mid-June to $166 \mathrm{~mm}$ in mid-December.

7. Gross examination of the subyearling migrants at John Day Dam and purse seine catches in the reservoir showed the fish to be in excellent condition. Preliminary analysis of stomach samples taken in 1982 and 1983 from purse seine catches demonstrated low numbers of empty stomachs and a high percentage of stomachs that were $\geq$ half full. This would tend to substantiate the hypothesis that large numbers of 0 -age chinook salmon are rearing in John Day Reservoir and may not be actively smolting.

8. The average residence time in John Day Reservoir for marked O-age chinook salmon released into the McNary Dam tailrace was 21 days.

9. Sixty percent of purse seine mark recaptures of O-age chinook salmon were made at or above the original release sites. 
10. Length of residence and the amount of upstream movement suggests large numbers of subyearling migrants in John Day Reservoir may not be actively migrating.

11. Regression analysis based on 15 data point 8 developed in 1983 provides no statistically significant evidence to lndicate that river flows are affecting the rates of downstream movement or residence times of 0 -age chinook salmon in John Day Reservoir. 


\section{ACKNOWLEDGEMENTS}

Support for this research came from the regions electrical ratepayers through Bonneville Power Administration. 
Miller, D. R. and C. W. Sims.

1983. Effects of flow on the migratory behavior and survival of juvenile fall and summer chinook salmon in John Day Reservoir. Natl. Mar. Fish. Serv., Annual Report of Research to Bonneville Power Administration, November 1983 (Contract DE-A179-81-BP27602). 25 p. plus Appendixes.

Sims, C. W., J. G. Williams, D. A. Faurot, R. C. Johnsen, and D. A. Brege. 1981. Migrational characteristics of juvenile salmon and steelhead in the Columbia River Basin and related passage research at John Day Dam, Volumes I and II. Natl. Mar. Fish. Serv., Report ot U.S. Army Corps of Engineers, May 1981 (Contracts DACW57-80-F-0394 and DACW68-78-C-0051). 61 p. plus Appendixes.

Sims, C. W., R. C. Johnsen, and D. A. Brege.

1982. Migrational characteristics of juvenile salmon and steelhead trout in the Columbia River system - 1981, Vol. 1 Assessment of the 1981 smolt migration. Natl. Mar. Fish. Serv.. Report to U.S. Army Corps of Engineers, April 1982 (Contracts DACW68-78-C-0051 and DACW57-81-F-0342). 16 p. plus Appendixes. 
APPENDIX A

BRAND RECAPTURE AND RIVER DISCHARGE DATA 
Appendix Table Al.--Brand recapture summary, O-age chinook salmon, John Day Dam (Turbine Unit 3), 1983.

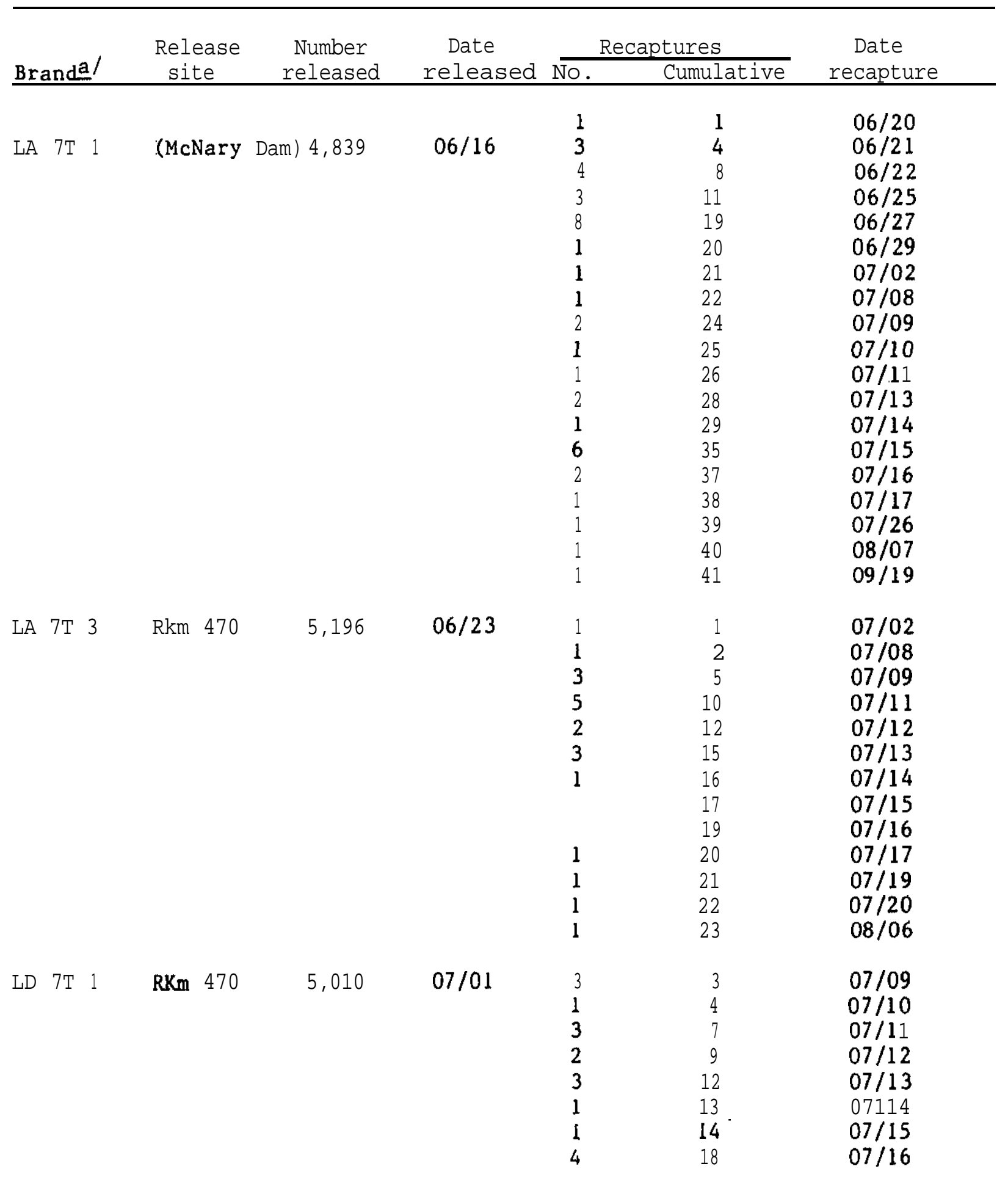


Appendix Table Al.--cont.

\begin{tabular}{|c|c|c|c|c|c|c|}
\hline \multirow{2}{*}{ Branda / } & \multirow{2}{*}{$\begin{array}{l}\text { Release } \\
\text { site }\end{array}$} & \multirow{2}{*}{$\begin{array}{c}\text { Number } \\
\text { released }\end{array}$} & \multirow{2}{*}{$\begin{array}{l}\text { Date } \\
\text { released }\end{array}$} & \multicolumn{2}{|c|}{ Recaptures } & \multirow{2}{*}{$\begin{array}{c}\text { Date } \\
\text { recapture }\end{array}$} \\
\hline & & & & No. & cumulatıve & \\
\hline & & & & 5 & 23 & $07 / 17$ \\
\hline & & & & 1 & 24 & $07 / 18$ \\
\hline & & & & 3 & 27 & $07 / 20$ \\
\hline & & & & 1 & 28 & $07 / 27$ \\
\hline \multirow[t]{17}{*}{ LA $2 \mathrm{~L} 1$} & $\mathrm{RKm} 470$ & 4,988 & $07 / 08$ & 1 & 1 & $07 / 16$ \\
\hline & & & & 3 & 4 & $07 / 17$ \\
\hline & & & & 3 & 7 & $07 / 18$ \\
\hline & & & & 3 & 10 & $07 / 19$ \\
\hline & & & & 5 & 15 & $07 / 20$ \\
\hline & & & & 2 & 17 & $07 / 21$ \\
\hline & & & & 2 & 19 & $07 / 22$ \\
\hline & & & & 3 & 22 & $07 / 23$ \\
\hline & & & & 1 & 23 & $07 / 26$ \\
\hline & & & & 3 & 26 & $07 / 27$ \\
\hline & & & & 2 & 28 & $07 / 30$ \\
\hline & & & & 1 & 29 & $08 / 01$ \\
\hline & & & & 1 & 30 & $08 / 08$ \\
\hline & & & & I & 31 & $08 / 23$ \\
\hline & & & & 1 & 32 & $08 / 30$ \\
\hline & & & & 2 & 34 & $09 / 09$ \\
\hline & & & & 1 & 35 & $11 / 28$ \\
\hline \multirow[t]{12}{*}{ LA 2L 3} & $\mathrm{RKm} 470$ & 5,005 & $07 / 13$ & 2 & 2 & $07 / 16$ \\
\hline & & & & 1 & 3 & $07 / 18$ \\
\hline & & & & 3 & 6 & $07 / 19$ \\
\hline & & & & 3 & 9 & $07 / 20$ \\
\hline & & & & 2 & 11 & $07 / 21$ \\
\hline & & & & 2 & 13 & $07 / 22$ \\
\hline & & & & & 14 & $07 / 26$ \\
\hline & & & & & 16 & $07 / 30$ \\
\hline & & & & 1 & 17 & $07 / 31$ \\
\hline & & & & 1 & 18 & $08 / 05$ \\
\hline & & & & 1 & 19 & $08 / 07$ \\
\hline & & & & 1 & 20 & $08 / 08$ \\
\hline \multirow[t]{9}{*}{ LD $2 \mathrm{~L} 1$} & $\mathrm{RKm}_{\mathrm{m}} 470$ & 5,014 & $07 / 15$ & I & 1 & $07 / 18$ \\
\hline & & & & 2 & 3 & $07 / 19$ \\
\hline & & & & 1 & 4 & $07 / 20$ \\
\hline & & & & 8 & 12 & $07 / 21$ \\
\hline & & & & 8 & 20 & $07 / 22$ \\
\hline & & & & 1 & 21 & $07 / 23$ \\
\hline & & & & 1 & 22 & $07 / 25$ \\
\hline & & & & 2 & 24 & $07 / 27$ \\
\hline & & & & 2 & 26 & $07 / 28$ \\
\hline
\end{tabular}


Appendix Table Al.--cont.

\begin{tabular}{|c|c|c|c|c|c|c|}
\hline \multirow[b]{2}{*}{ Bran\&/ } & \multirow{2}{*}{$\begin{array}{c}\text { Release } \\
\text { site }\end{array}$} & \multirow{2}{*}{$\begin{array}{c}\text { Number } \\
\text { released }\end{array}$} & \multirow{2}{*}{$\begin{array}{c}\text { Date } \\
\text { released }\end{array}$} & \multicolumn{2}{|c|}{ Recaptures } & \multirow{2}{*}{$\begin{array}{c}\text { Date } \\
\text { recapture }\end{array}$} \\
\hline & & & & No. & Cumulative & \\
\hline & & & & $\begin{array}{l}1 \\
1 \\
1 \\
1 \\
1 \\
1 \\
1 \\
1 \\
1 \\
1 \\
1 \\
1 \\
1 \\
1 \\
1 \\
1\end{array}$ & $\begin{array}{l}27 \\
28 \\
29 \\
30 \\
31 \\
32 \\
33 \\
34 \\
35 \\
36 \\
37 \\
38 \\
39 \\
40 \\
41 \\
42\end{array}$ & $\begin{array}{l}07 / 29 \\
07 / 30 \\
07 / 31 \\
08 / 01 \\
08 / 02 \\
08 / 03 \\
08 / 05 \\
08 / 07 \\
08 / 08 \\
08 / 11 \\
08 / 12 \\
08 / 17 \\
09 / 05 \\
09 / 11 \\
10 / 06 \\
12 / 05\end{array}$ \\
\hline $\begin{array}{lll}\mathrm{LA} & 2 \mathrm{~T} & 1\end{array}$ & RKm 470 & 5,019 & $07 / 20$ & $\begin{array}{l}1 \\
4 \\
1 \\
3 \\
4 \\
4 \\
I \\
3 \\
1 \\
7 \\
2 \\
2 \\
1 \\
1 \\
2 \\
2 \\
1 \\
1 \\
1 \\
1 \\
1 \\
1 \\
1 \\
2 \\
1 \\
1 \\
1 \\
2 \\
1\end{array}$ & $\begin{array}{r}1 \\
5 \\
6 \\
9 \\
13 \\
17 \\
18 \\
21 \\
22 \\
29 \\
31 \\
33 \\
34 \\
35 \\
37 \\
39 \\
40 \\
41 \\
42 \\
43 \\
44 \\
45 \\
46 \\
48 \\
49 \\
50 \\
51 \\
53 \\
54\end{array}$ & $\begin{array}{l}07 / 24 \\
07 / 25 \\
07 / 27 \\
07 / 28 \\
07 / 29 \\
07 / 30 \\
07 / 31 \\
08 / 01 \\
08 / 03 \\
08 / 08 \\
08 / 09 \\
08 / 10 \\
08 / 12 \\
08 / 14 \\
08 / 19 \\
08 / 24 \\
08 / 27 \\
08 / 28 \\
08 / 29 \\
08 / 30 \\
08 / 31 \\
09 / 01 \\
09 / 02 \\
09 / 05 \\
09 / 07 \\
09 / 08 \\
09 / 09 \\
09 / 11 \\
09 / 16\end{array}$ \\
\hline
\end{tabular}


Appendix Table Al.--cont.

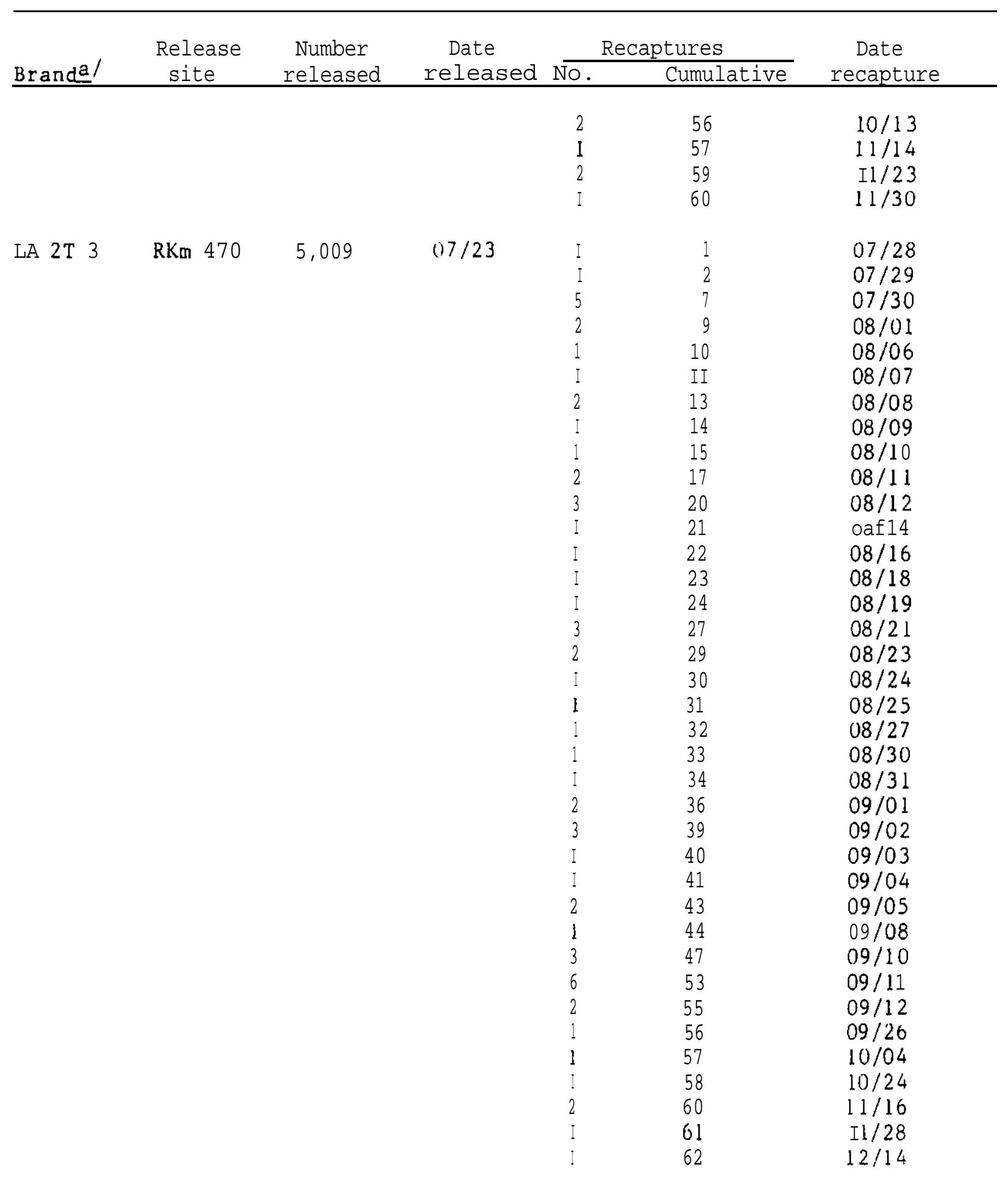


Appendix Table Al.--Cont.

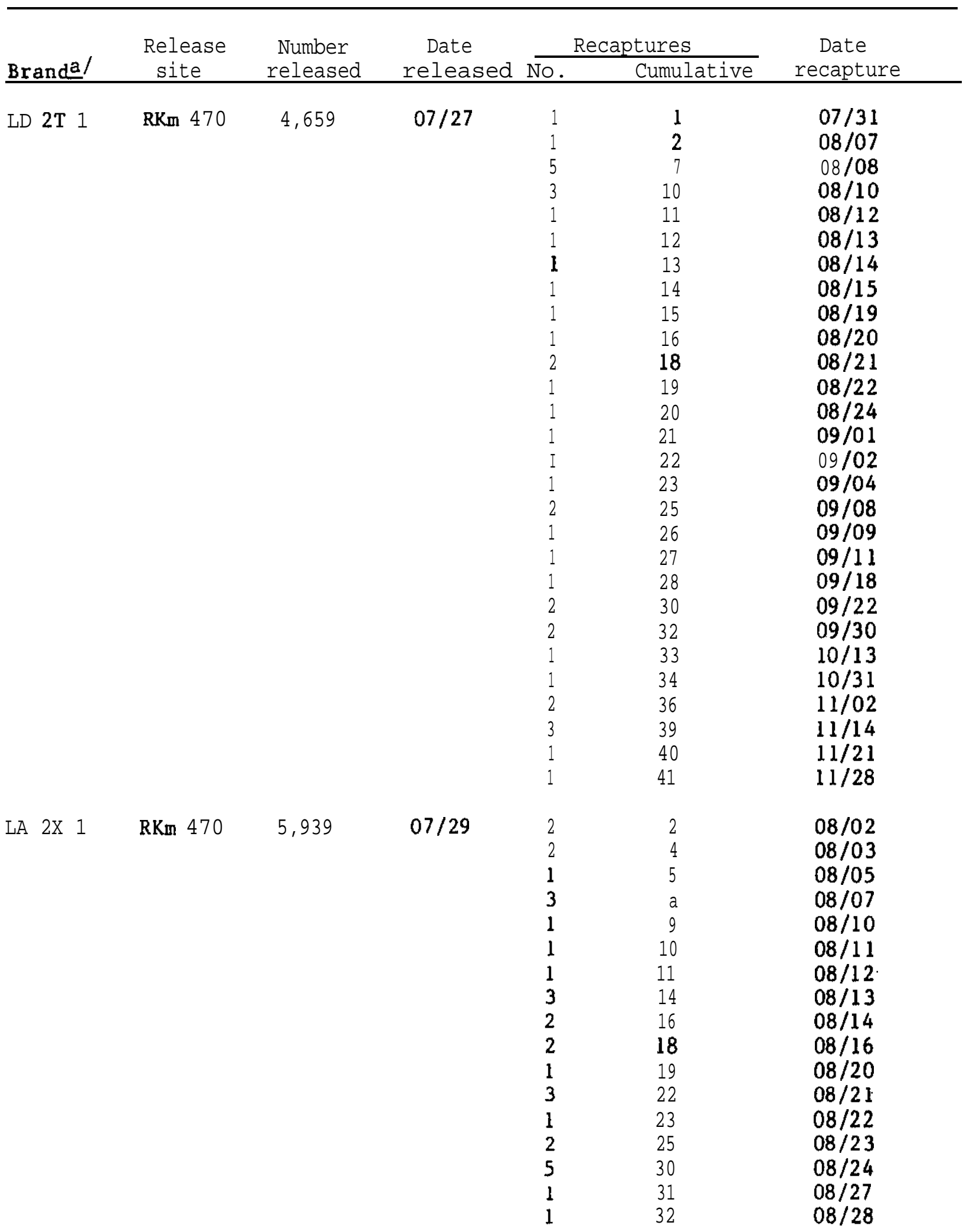


Appendix Table Al .--cont.

\begin{tabular}{|c|c|c|c|c|c|c|}
\hline Branda/ & $\begin{array}{l}\text { Release } \\
\text { site }\end{array}$ & $\begin{array}{l}\text { Number } \\
\text { released }\end{array}$ & $\begin{array}{l}\text { Date } \\
\text { released } \\
\end{array}$ & $\mathrm{NO}$ & $\begin{array}{l}\text { ptures } \\
\text { Cumulative }\end{array}$ & $\begin{array}{l}\text { Date } \\
\text { recapture }\end{array}$ \\
\hline & & & & $\begin{array}{l}1 \\
1 \\
1 \\
3 \\
1 \\
1 \\
3 \\
3 \\
7 \\
1 \\
1 \\
1 \\
1 \\
1 \\
1 \\
1 \\
1 \\
1 \\
2 \\
1 \\
1 \\
1 \\
1 \\
1 \\
2\end{array}$ & $\begin{array}{l}33 \\
34 \\
35 \\
38 \\
39 \\
40 \\
43 \\
46 \\
53 \\
54 \\
55 \\
56 \\
57 \\
58 \\
59 \\
60 \\
61 \\
62 \\
64 \\
65 \\
66 \\
67 \\
68 \\
69 \\
71\end{array}$ & $\begin{array}{l}\text { oaf } 29 \\
08 / 30 \\
08 / 31 \\
09 / 05 \\
09 / 07 \\
09 / 08 \\
09 / 09 \\
09 / 10 \\
09 / 11 \\
09 / 12 \\
09 / 17 \\
09 / 18 \\
09 / 19 \\
09 / 20 \\
10 / 04 \\
10 / 05 \\
10 / 17 \\
10 / 19 \\
10 / 20 \\
10 / 21 \\
11 / 04 \\
11 / 11 \\
11 / 23 \\
11 / 24 \\
12 / 02\end{array}$ \\
\hline LA $2 \times 3$ & RKm 470 & 4,657 & $08 / 05$ & $\begin{array}{l}\mathrm{I} \\
1 \\
5 \\
4 \\
3 \\
1 \\
1 \\
1 \\
2 \\
1 \\
2 \\
3 \\
2 \\
1 \\
2 \\
2 \\
1 \\
1 \\
2 \\
I\end{array}$ & $\begin{array}{r}1 \\
2 \\
7 \\
11 \\
14 \\
15 \\
16 \\
17 \\
19 \\
20 \\
22 \\
25 \\
27 \\
28 \\
30 \\
32 \\
33 \\
34 \\
36 \\
37\end{array}$ & $\begin{array}{l}08 / 09 \\
08 / 10 \\
08 / 11 \\
08 / 12 \\
08 / 13 \\
08 / 15 \\
08 / 18 \\
08 / 19 \\
08 / 20 \\
08 / 22 \\
08 / 23 \\
08 / 24 \\
08 / 29 \\
09 / 01 \\
09 / 02 \\
09 / 05 \\
09 / 07 \\
09 / 08 \\
09 / 09 \\
09 / 10\end{array}$ \\
\hline
\end{tabular}


Appendix Table Al.--cont.

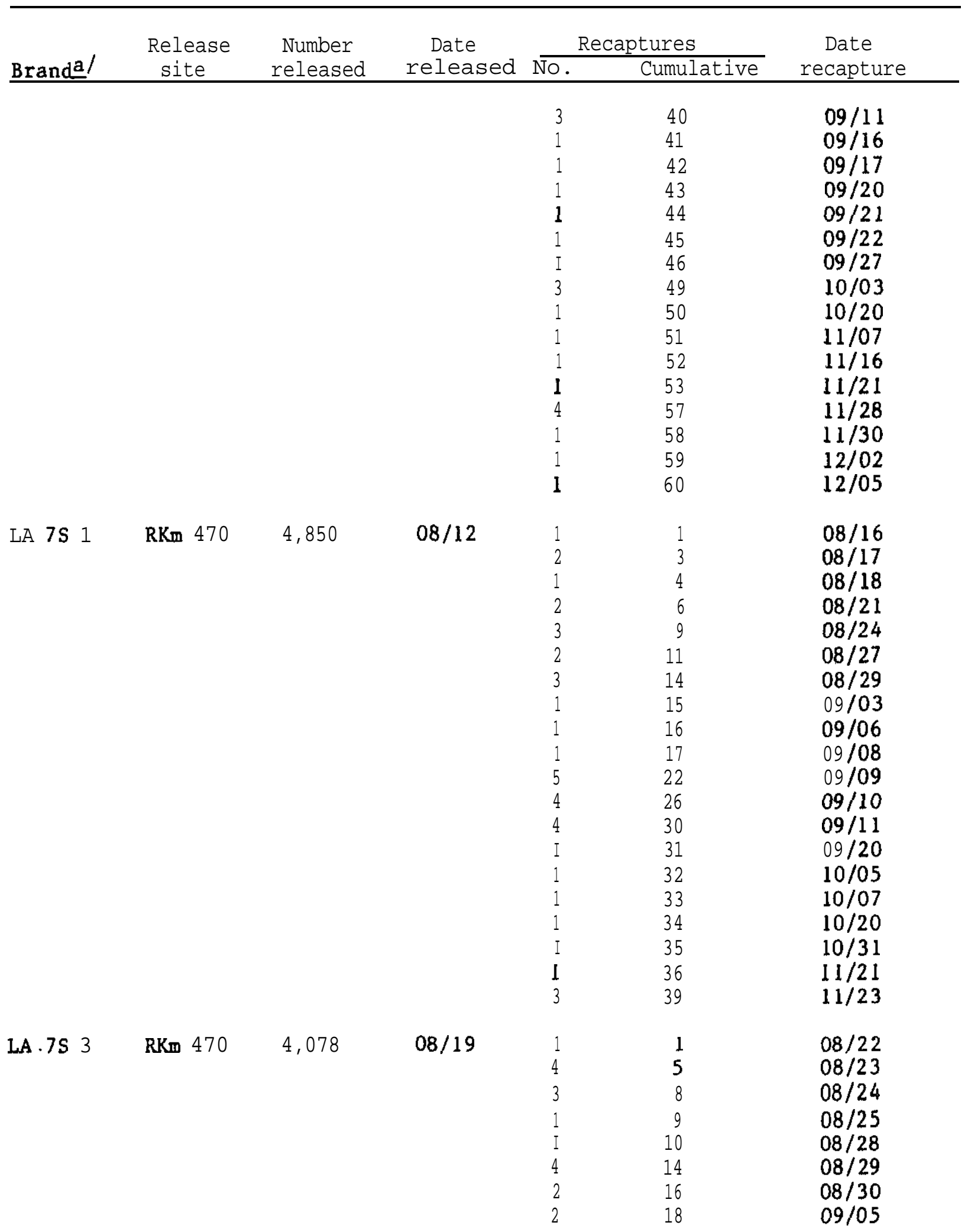


Appendix Table Al .--cont.

\begin{tabular}{|c|c|c|c|c|c|c|}
\hline \multirow[b]{2}{*}{ Bran\&f } & \multirow{2}{*}{$\begin{array}{c}\text { Release } \\
\text { site }\end{array}$} & \multirow{2}{*}{$\begin{array}{l}\text { Number } \\
\text { released }\end{array}$} & \multirow{2}{*}{$\begin{array}{l}\text { Date } \\
\text { released }\end{array}$} & \multicolumn{2}{|c|}{ Recaptures } & \multirow{2}{*}{$\begin{array}{l}\text { Date } \\
\text { recapture }\end{array}$} \\
\hline & & & & No. & Cumulative & \\
\hline & & & & $\begin{array}{l}1 \\
I \\
I \\
5 \\
2 \\
I \\
2 \\
1 \\
3 \\
1 \\
1 \\
2 \\
1 \\
1 \\
I \\
2 \\
1 \\
2\end{array}$ & $\begin{array}{l}19 \\
20 \\
21 \\
26 \\
28 \\
29 \\
31 \\
32 \\
35 \\
36 \\
37 \\
39 \\
40 \\
41 \\
42 \\
44 \\
45 \\
47\end{array}$ & $\begin{array}{l}09 / 06 \\
09 / 08 \\
09 / 10 \\
09 / 11 \\
09 / 12 \\
09 / 13 \\
09 / 16 \\
09 / 17 \\
09 / 18 \\
09 / 19 \\
09 / 21 \\
09 / 22 \\
10 / 03 \\
10 / 31 \\
11 / 07 \\
11 / 16 \\
11 / 28 \\
12 / 02\end{array}$ \\
\hline LD 7S 1 & RKm 470 & 5,641 & $08 / 26$ & $\begin{array}{l}1 \\
1 \\
1 \\
1 \\
2 \\
2 \\
1 \\
2 \\
1\end{array}$ & $\begin{array}{r}1 \\
5 \\
9 \\
12 \\
13 \\
18 \\
20 \\
22 \\
23 \\
24 \\
26 \\
27 \\
28 \\
29 \\
31 \\
32 \\
33 \\
35 \\
36 \\
37 \\
38 \\
39 \\
41 \\
43 \\
44 \\
46 \\
47\end{array}$ & $\begin{array}{l}08 / 30 \\
0 a f 31 \\
09 / 01 \\
09 / 02 \\
09 / 03 \\
09 / 05 \\
09 / 06 \\
09 / 07 \\
09 / 08 \\
09 / 09 \\
09 / 10 \\
09 / 11 \\
09 / 12 \\
09 / 16 \\
09 / 18 \\
09 / 19 \\
09 / 21 \\
09 / 22 \\
09 / 26 \\
09 / 30 \\
10 / 17 \\
10 / 20 \\
10 / 31 \\
11 / 07 \\
11 / 11 \\
11 / 14 \\
11 / 16\end{array}$ \\
\hline
\end{tabular}


Appendix Table Al.--cont.

\begin{tabular}{|c|c|c|c|c|c|c|}
\hline \multirow[b]{2}{*}{ Branda/ } & \multirow{2}{*}{$\begin{array}{c}\text { Release } \\
\text { site }\end{array}$} & \multirow{2}{*}{$\begin{array}{c}\text { Number } \\
\text { released }\end{array}$} & \multirow{2}{*}{$\begin{array}{l}\text { Date } \\
\text { released }\end{array}$} & \multicolumn{2}{|c|}{ Recaptures } & \multirow{2}{*}{$\begin{array}{l}\text { Date } \\
\text { recapture }\end{array}$} \\
\hline & & & & No. & Cumulative & \\
\hline & & & & $\begin{array}{l}3 \\
1 \\
1 \\
1 \\
1\end{array}$ & $\begin{array}{l}50 \\
51 \\
52 \\
53 \\
54\end{array}$ & $\begin{array}{l}11 / 18 \\
11 / 28 \\
11 / 30 \\
12 / 02 \\
12 / 07\end{array}$ \\
\hline LD $7 S 3$ & $\mathrm{RKm} 470$ & 1,855 & $09 / 02$ & $\begin{array}{l}3 \\
2 \\
6 \\
1 \\
1 \\
1 \\
1 \\
1 \\
1\end{array}$ & $\begin{array}{r}3 \\
5 \\
11 \\
12 \\
13 \\
14 \\
15 \\
16 \\
17\end{array}$ & $\begin{array}{l}09 / 08 \\
09 / 09 \\
09 / 11 \\
09 / 12 \\
10 / 06 \\
10 / 12 \\
10 / 31 \\
11 / 11 \\
11 / 16\end{array}$ \\
\hline LA E 4 & $\begin{array}{l}\text { RKm } 373 \\
\text { (purse }\end{array}$ & $n e)^{363}$ & $07 / 06$ & 1 & 1 & $07 / 10$ \\
\hline LA E 3 & $\mathrm{RKm} 348$ & 534 & $07 / 07$ & $\begin{array}{l}8 \\
1\end{array}$ & $\begin{array}{l}a \\
9\end{array}$ & $\begin{array}{l}07 / 08 \\
07 / 09\end{array}$ \\
\hline LA E 2 & RKm 407 & 1,108 & $07 / 12$ & $\begin{array}{l}1 \\
2\end{array}$ & $\begin{array}{l}1 \\
3\end{array}$ & $\begin{array}{l}07 / 19 \\
07 / 23\end{array}$ \\
\hline LA E 1 & RKm 430 & 1,655 & $07 / 13$ & $\begin{array}{l}3 \\
1\end{array}$ & $\begin{array}{l}3 \\
4\end{array}$ & $\begin{array}{l}07 / 21 \\
07 / 28\end{array}$ \\
\hline LD E I & RKm 389 & 1,786 & $07 / 14$ & $\begin{array}{l}2 \\
1 \\
1 \\
1 \\
1\end{array}$ & $\begin{array}{l}2 \\
3 \\
4 \\
5 \\
6\end{array}$ & $\begin{array}{l}07 / 19 \\
07 / 20 \\
07 / 27 \\
08 / 16 \\
09 / 10\end{array}$ \\
\hline LD E 2 & RKm 357 & 979 & $07 / 20$ & $\begin{array}{l}2 \\
1\end{array}$ & $\begin{array}{l}2 \\
3\end{array}$ & $\begin{array}{l}07 / 21 \\
07 / 22\end{array}$ \\
\hline LD E 3 & RKm 348 & 1,502 & $07 / 21$ & $\begin{array}{l}7 \\
4 \\
5 \\
1 \\
1\end{array}$ & $\begin{array}{r}7 \\
11 \\
16 \\
17 \\
1 a\end{array}$ & $\begin{array}{l}07 / 22 \\
07 / 23 \\
07 / 24 \\
07 / 30 \\
08 / 13\end{array}$ \\
\hline LA HE 1 & $\mathbf{R K m} 407$ & 735 & $07 / 26$ & $\begin{array}{l}1 \\
1\end{array}$ & $\begin{array}{l}1 \\
2\end{array}$ & $\begin{array}{l}08 / 01 \\
09 / 10\end{array}$ \\
\hline
\end{tabular}


Appendix Table Al ---cont .

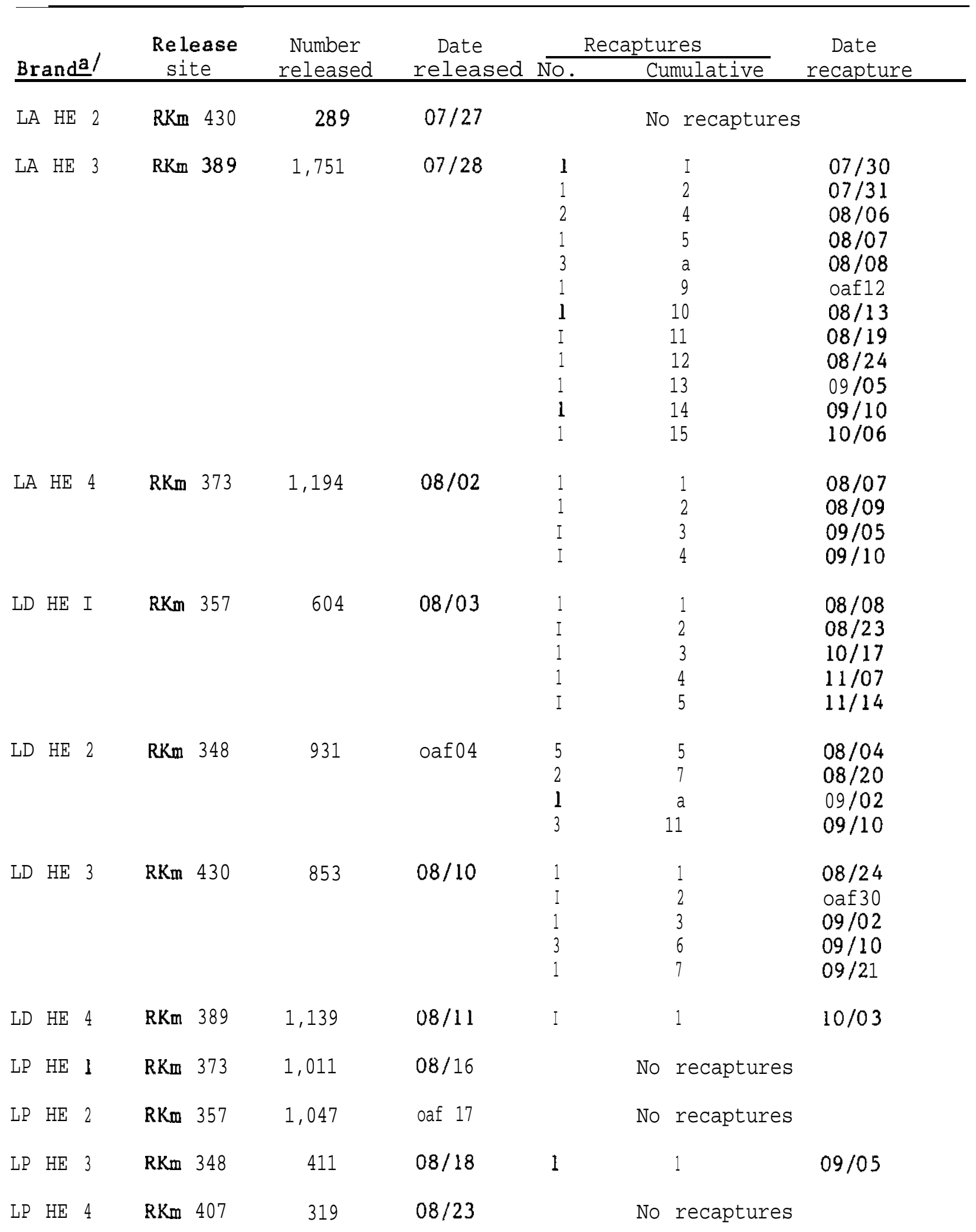


Appendix Table Al.--cunt.

\begin{tabular}{|c|c|c|c|c|c|c|c|}
\hline \multirow{2}{*}{ Branda/ } & \multirow{2}{*}{$\begin{array}{l}\text { Release } \\
\text { site }\end{array}$} & \multirow{2}{*}{$\begin{array}{c}\text { Number } \\
\text { released }\end{array}$} & \multirow{2}{*}{$\begin{array}{l}\text { Date } \\
\text { released }\end{array}$} & \multicolumn{3}{|c|}{ Recaptures } & \multirow{2}{*}{$\begin{array}{l}\text { Date } \\
\text { recapture }\end{array}$} \\
\hline & & & & No. & & ulative & \\
\hline RA HE 1 & RKm 430 & 788 & $08 / 24$ & & No & recaptures & \\
\hline $\mathrm{RA} \quad \mathrm{HE} 2$ & $\mathrm{RKm} 348$ & 305 & $09 / 01$ & 1 & & 1 & $10 / 04$ \\
\hline $\mathrm{RA} \quad \mathrm{HE} 3$ & RKm 407 & 572 & $09 / 06$ & $\begin{array}{l}1 \\
1\end{array}$ & & $\begin{array}{l}1 \\
2\end{array}$ & $\begin{array}{l}09 / 19 \\
09 / 26\end{array}$ \\
\hline RA HE 4 & RKm 348 & 552 & $09 / 07$ & & No & recaptures & \\
\hline RD $\mathrm{HE} \quad 1$ & RKm 389 & 194 & $09 / 08$ & 1 & & 1 & $11 / 14$ \\
\hline $\mathrm{RD}$ HE 2 & RKm 373 & 280 & $09 / 13$ & & No & recaptures & \\
\hline $\mathrm{RD} \mathrm{HE} 3$ & RKm 357 & 170 & $09 / 14$ & $\begin{array}{l}1 \\
1\end{array}$ & & $\begin{array}{l}1 \\
2\end{array}$ & $\begin{array}{l}10 / 31 \\
11 / 28\end{array}$ \\
\hline RDHF . 4 & $\mathrm{RKm} 348$ & 39 & $09 / 15$ & & No & recaptures & \\
\hline RP HE 1 & RKm 407 & 171 & $09 / 20$ & 1 & & 1 & $10 / 19$ \\
\hline $\mathrm{RP} \quad \mathrm{HE} 2$ & RKm 430 & 146 & $09 / 21$ & & No & recaptures & \\
\hline $\mathrm{RP} \quad \mathrm{HE} 3$ & RKm 389 & 213 & $09 / 22$ & & No & recaptures & \\
\hline $\mathrm{RP} \quad \mathrm{HE} \quad 4$ & RKm 357 & 62 & $09 / 28$ & 1 & & 1 & $10 / 03$ \\
\hline LA D I & rub 348 & 103 & $09 / 29$ & & No & recaptures & \\
\hline
\end{tabular}


Appendix Table A2.--Brand recapture summary--purse seine catches of 0-age chinook salmon in John Day Reservoir, 1983.

\begin{tabular}{|c|c|c|c|c|c|c|c|}
\hline \multirow{2}{*}{ Branda/ } & \multirow{2}{*}{$\begin{array}{l}\text { Release } \\
\text { site }\end{array}$} & \multirow{2}{*}{$\begin{array}{l}\text { Number } \\
\text { released }\end{array}$} & \multirow{2}{*}{$\begin{array}{l}\text { Date } \\
\text { released }\end{array}$} & \multicolumn{2}{|c|}{ Recaptures } & \multirow{2}{*}{$\begin{array}{l}\text { Date } \\
\text { recapture }\end{array}$} & \multirow{2}{*}{$\begin{array}{r}\text { Recapture } \\
\text { site (Rkm) }\end{array}$} \\
\hline & & & & No. & Cumulative & & \\
\hline \multirow[t]{7}{*}{ LA $7 \mathrm{~T} 1$} & \multirow{7}{*}{\multicolumn{2}{|c|}{ (McNary Dam) }} & $06 / 16$ & $\begin{array}{l}2 \\
3\end{array}$ & $\begin{array}{l}2 \\
5\end{array}$ & $\begin{array}{l}07 / 06 \\
07 / 07\end{array}$ & $\begin{array}{l}373 \\
348\end{array}$ \\
\hline & & & & 2 & 7 & $07 / 12$ & 407 \\
\hline & & & & 3 & 10 & $07 / 13$ & 430 \\
\hline & & & & 1 & 11 & $07 / 14$ & 389 \\
\hline & & & & 1 & 12 & $07 / 20$ & 357 \\
\hline & & & & 3 & 15 & $07 / 21$ & 348 \\
\hline & & & & 1 & 16 & $07 / 28$ & 389 \\
\hline \multirow[t]{10}{*}{ LA $7 \mathrm{~T} 3$} & \multirow[t]{10}{*}{ RKm 470} & \multirow[t]{10}{*}{5,196} & \multirow[t]{10}{*}{$06 / 23$} & 2 & 2 & $07 / 06$ & 373 \\
\hline & & & & 3 & 5 & $07 / 07$ & 348 \\
\hline & & & & 2 & 7 & $07 / 12$ & 407 \\
\hline & & & & 1 & a & $07 / 13$ & 430 \\
\hline & & & & 1 & 9 & $07 / 14$ & 389 \\
\hline & & & & 2 & 11 & $07 / 20$ & 357 \\
\hline & & & & 3 & 14 & $07 / 21$ & 348 \\
\hline & & & & 1 & 15 & $07 / 26$ & 407 \\
\hline & & & & 1 & 16 & $07 / 28$ & 389 \\
\hline & & & & 1 & 17 & $08 / 03$ & 357 \\
\hline \multirow[t]{9}{*}{$\mathrm{LD} 7 \mathrm{~T} \mathrm{I}$} & \multirow[t]{9}{*}{$\mathrm{kKm} 470$} & \multirow[t]{9}{*}{5,010} & \multirow[t]{9}{*}{$07 / 01$} & 3 & 3 & $07 / 12$ & 407 \\
\hline & & & & 3 & 6 & $07 / 13$ & 430 \\
\hline & & & & 5 & 11 & $07 / 14$ & 389 \\
\hline & & & & 1 & 12 & $07 / 20$ & 357 \\
\hline & & & & 2 & 14 & $07 / 21$ & 348 \\
\hline & & & & 3 & 17 & $07 / 26$ & 407 \\
\hline & & & & I & la & $08 / 02$ & 373 \\
\hline & & & & 1 & 19 & oaf 10 & 430 \\
\hline & & & & I & 20 & $08 / 11$ & 389 \\
\hline \multirow[t]{10}{*}{ LA 2L 1} & \multirow[t]{10}{*}{$\mathrm{RKm} 470$} & \multirow[t]{10}{*}{4,988} & \multirow[t]{10}{*}{$07 / 08$} & 4 & 4 & $07 / 12$ & 407 \\
\hline & & & & 6 & 10 & $07 / 13$ & 430 \\
\hline & & & & 5 & 15 & $07 / 14$ & 389 \\
\hline & & & & 1 & 16 & $07 / 20$ & 357 \\
\hline & & & & 2 & la & $07 / 21$ & 348 \\
\hline & & & & 2 & 20 & $07 / 28$ & 389 \\
\hline & & & & I & 21 & $08 / 16$ & 373 \\
\hline & & & & 2 & 23 & $08 / 24$ & 430 \\
\hline & & & & I & 24 & $09 / 06$ & 407 \\
\hline & & & & I & 25 & $09 / 08$ & 389 \\
\hline
\end{tabular}


Appendix Table A2.--cont.

\begin{tabular}{|c|c|c|c|c|c|c|c|}
\hline \multirow[b]{2}{*}{ Bran\&/ } & \multirow{2}{*}{$\begin{array}{c}\text { Release } \\
\text { site }\end{array}$} & \multirow{2}{*}{$\begin{array}{c}\text { Number } \\
\text { released }\end{array}$} & \multirow{2}{*}{$\begin{array}{c}\text { Date } \\
\text { released } \\
\end{array}$} & \multicolumn{2}{|c|}{ Recaptures } & \multirow{2}{*}{$\begin{array}{c}\text { Date } \\
\text { recapture }\end{array}$} & \multirow{2}{*}{$\begin{array}{r}\text { Recapture } \\
\text { site (Rkm) }\end{array}$} \\
\hline & & & & No. & Cumulative & & \\
\hline \multirow[t]{12}{*}{ LA $2 \mathrm{~L} 3$} & $\mathrm{RKm} 470$ & 5,005 & $07 / 13$ & 4 & 4 & $07 / 14$ & 389 \\
\hline & & & & 2 & 6 & $07 / 20$ & 357 \\
\hline & & & & 2 & 8 & $07 / 21$ & 348 \\
\hline & & & & 3 & II & $07 / 26$ & 407 \\
\hline & & & & 3 & 14 & $08 / 16$ & 373 \\
\hline & & & & 1 & 15 & $08 / 18$ & 348 \\
\hline & & & & 1 & 16 & $08 / 23$ & 407 \\
\hline & & & & 1 & 17 & $08 / 24$ & 430 \\
\hline & & & & 1 & la & $09 / 01$ & 348 \\
\hline & & & & 2 & 20 & $09 / 06$ & 407 \\
\hline & & & & 1 & 21 & $09 / 07$ & 430 \\
\hline & & & & 1 & 22 & $09 / 15$ & 348 \\
\hline \multirow[t]{16}{*}{ LD $2 \mathrm{~L} 1$} & RKm 470 & 5,014 & $07 / 15$ & 3 & 3 & $07 / 20$ & 357 \\
\hline & & & & 9 & 12 & $07 / 21$ & 348 \\
\hline & & & & 3 & 15 & $07 / 26$ & 407 \\
\hline & & & & a & 23 & $07 / 28$ & 389 \\
\hline & & & & 2 & 25 & $08 / 02$ & 373 \\
\hline & & & & 2 & 27 & $08 / 03$ & 357 \\
\hline & & & & 2 & 29 & $08 / 04$ & 348 \\
\hline & & & & 2 & 31 & $08 / 10$ & 430 \\
\hline & & & & 1 & 32 & $08 / 11$ & 389 \\
\hline & & & & 1 & 33 & $08 / 17$ & 373 \\
\hline & & & & 3 & 36 & $08 / 17$ & 357 \\
\hline & & & & 1 & 37 & $09 / 06$ & 407 \\
\hline & & & & 1 & 38 & $09 / 07$ & 430 \\
\hline & & & & 1 & 39 & $09 / 08$ & 389 \\
\hline & & & & 1 & 40 & $09 / 20$ & 407 \\
\hline & & & & 1 & 41 & $09 / 22$ & 389 \\
\hline \multirow[t]{15}{*}{ LA $2 \mathrm{~T} \mathrm{I}$} & $\mathrm{RKm} 470$ & 5,019 & $07 / 20$ & 2 & 2 & $07 / 26$ & 407 \\
\hline & & & & 3 & 5 & $07 / 27$ & 430 \\
\hline & & & & 5 & 10 & $07 / 28$ & 389 \\
\hline & & & & 1 & 11 & $08 / 02$ & 373 \\
\hline & & & & 2 & 13 & $08 / 03$ & 357 \\
\hline & & & & 3 & 16 & $08 / 04$ & 348 \\
\hline & & & & 3 & 19 & $08 / 16$ & 373 \\
\hline & & & & 3 & 22 & $08 / 17$ & 357 \\
\hline & & & & 3 & 25 & $08 / 23$ & 348 \\
\hline & & & & 3 & 28 & $08 / 24$ & 430 \\
\hline & & & & 1 & 29 & $09 / 06$ & 407 \\
\hline & & & & 1 & 30 & $09 / 07$ & 430 \\
\hline & & & & 1 & 31 & $09 / 13$ & 373 \\
\hline & & & & 1 & 32 & $09 / 20$ & 407 \\
\hline & & & & 1 & 33 & $09 / 22$ & 389 \\
\hline
\end{tabular}


Appendix Table A2.--cont .

\begin{tabular}{|c|c|c|c|c|c|c|c|}
\hline \multirow[b]{2}{*}{ Bran\&f } & \multirow{2}{*}{$\begin{array}{l}\text { Release } \\
\text { site }\end{array}$} & \multirow{2}{*}{$\begin{array}{l}\text { Number } \\
\text { released }\end{array}$} & \multirow{2}{*}{$\begin{array}{l}\text { Date } \\
\text { released }\end{array}$} & \multicolumn{2}{|c|}{ Recaptures } & \multirow{2}{*}{$\begin{array}{c}\text { Date } \\
\text { recapture }\end{array}$} & \multirow{2}{*}{$\begin{array}{r}\text { Recapture } \\
\text { site (Rkm) }\end{array}$} \\
\hline & & & & $\overline{\mathrm{No}}$ & Cumulative & & \\
\hline LA $2 \mathrm{~T} 3$ & $\mathrm{RKm} 470$ & 5,009 & $07 / 23$ & $\begin{array}{l}5 \\
2 \\
5 \\
1 \\
3 \\
1 \\
2 \\
1 \\
2 \\
1 \\
3 \\
1 \\
1\end{array}$ & $\begin{array}{r}5 \\
7 \\
12 \\
13 \\
16 \\
17 \\
19 \\
20 \\
22 \\
23 \\
26 \\
27 \\
28 \\
29 \\
30 \\
31 \\
32\end{array}$ & $\begin{array}{l}07 / 26 \\
07 / 27 \\
07 / 28 \\
08 / 02 \\
08 / 03 \\
08 / 04 \\
08 / 10 \\
08 / 11 \\
08 / 16 \\
08 / 17 \\
08 / 18 \\
08 / 23 \\
09 / 06 \\
09 / 08 \\
09 / 14 \\
09 / 20 \\
09 / 28\end{array}$ & $\begin{array}{l}407 \\
430 \\
389 \\
373 \\
357 \\
348 \\
430 \\
389 \\
373 \\
357 \\
348 \\
407 \\
407 \\
389 \\
357 \\
407 \\
357\end{array}$ \\
\hline $\mathrm{LD} 2 \mathrm{~T} \mathbf{l}$ & $\mathrm{RKm} 470$ & 4,659 & $07 / 27$ & $\begin{array}{l}3 \\
4 \\
1 \\
3 \\
1 \\
4 \\
2 \\
2 \\
2 \\
1\end{array}$ & $\begin{array}{r}3 \\
7 \\
8 \\
11 \\
12 \\
16 \\
18 \\
20 \\
22 \\
23\end{array}$ & $\begin{array}{l}08 / 02 \\
08 / 04 \\
08 / 11 \\
08 / 16 \\
08 / 17 \\
08 / 24 \\
09 / 06 \\
09 / 07 \\
09 / 08 \\
09 / 13\end{array}$ & $\begin{array}{l}373 \\
348 \\
389 \\
373 \\
357 \\
430 \\
407 \\
430 \\
389 \\
373\end{array}$ \\
\hline LA $2 X \quad \mathrm{I}$ & RKm 470 & 5,939 & $07 / 29$ & $\begin{array}{l}3 \\
3 \\
3 \\
1 \\
3 \\
2 \\
4 \\
I \\
3 \\
I \\
I \\
2\end{array}$ & $\begin{array}{r}3 \\
6 \\
9 \\
10 \\
13 \\
15 \\
19 \\
20 \\
23 \\
24 \\
25 \\
27\end{array}$ & $\begin{array}{l}08 / 02 \\
\text { oaf04 } \\
08 / 10 \\
08 / 11 \\
08 / 16 \\
\text { oaf17 } \\
08 / 24 \\
09 / 01 \\
09 / 06 \\
09 / 07 \\
09 / 15 \\
09 / 29\end{array}$ & $\begin{array}{l}373 \\
348 \\
430 \\
389 \\
373 \\
357 \\
430 \\
348 \\
407 \\
430 \\
348 \\
348\end{array}$ \\
\hline
\end{tabular}


Appendix Table A2.---cont .

\begin{tabular}{|c|c|c|c|c|c|c|c|}
\hline \multirow[b]{2}{*}{ Branda/ } & \multirow{2}{*}{$\begin{array}{l}\text { Release } \\
\text { site }\end{array}$} & \multirow{2}{*}{$\begin{array}{c}\text { Number } \\
\text { released }\end{array}$} & \multirow{2}{*}{$\begin{array}{l}\text { Date } \\
\text { rel eased } \\
\end{array}$} & \multicolumn{2}{|c|}{ Recaptures } & \multirow{2}{*}{$\begin{array}{l}\text { Date } \\
\text { recapture }\end{array}$} & \multirow{2}{*}{$\begin{array}{r}\text { Recapture } \\
\text { site ( } \mathbf{R k m})\end{array}$} \\
\hline & & & & No. & Cumulative & & \\
\hline LA $2 \times 3$ & RKm 470 & 4,657 & oaf05 & $\begin{array}{l}3 \\
4 \\
3 \\
7 \\
2 \\
1 \\
3 \\
1 \\
2 \\
1 \\
1 \\
1 \\
1\end{array}$ & $\begin{array}{r}3 \\
7 \\
10 \\
17 \\
19 \\
20 \\
23 \\
24 \\
26 \\
27 \\
28 \\
29 \\
30\end{array}$ & $\begin{array}{l}08 / 10 \\
08 / 11 \\
08 / 16 \\
08 / 17 \\
08 / 18 \\
08 / 23 \\
08 / 24 \\
09 / 06 \\
09 / 07 \\
09 / 08 \\
09 / 21 \\
09 / 22 \\
09 / 29\end{array}$ & $\begin{array}{l}430 \\
389 \\
373 \\
357 \\
348 \\
407 \\
430 \\
407 \\
430 \\
389 \\
430 \\
389 \\
348\end{array}$ \\
\hline LA 7s I & $\mathrm{RKm} 470$ & 4,850 & $08 / 12$ & $\begin{array}{l}3 \\
1 \\
3 \\
3 \\
4 \\
1 \\
3 \\
1 \\
1 \\
1\end{array}$ & $\begin{array}{l}3 \\
4 \\
7 \\
10 \\
14 \\
15 \\
1 a \\
19 \\
20 \\
21\end{array}$ & $\begin{array}{l}08 / 16 \\
08 / 17 \\
08 / 18 \\
08 / 23 \\
08 / 24 \\
09 / 01 \\
09 / 06 \\
09 / 07 \\
09 / 08 \\
09 / 22\end{array}$ & $\begin{array}{l}373 \\
357 \\
348 \\
407 \\
430 \\
348 \\
407 \\
430 \\
389 \\
389\end{array}$ \\
\hline LA 7S 3 & $\mathrm{RKm} 470$ & 4,878 & $08 / 19$ & $\begin{array}{l}1 \\
1 \\
2 \\
1 \\
2 \\
1 \\
1 \\
1\end{array}$ & $\begin{array}{r}1 \\
2 \\
4 \\
5 \\
7 \\
a \\
9 \\
10\end{array}$ & $\begin{array}{l}08 / 23 \\
08 / 24 \\
09 / 01 \\
09 / 07 \\
09 / 08 \\
09 / 13 \\
09 / 20 \\
09 / 21\end{array}$ & $\begin{array}{l}407 \\
430 \\
348 \\
430 \\
389 \\
373 \\
407 \\
430\end{array}$ \\
\hline LD 7S I & $\mathrm{RKm} 470$ & 5,641 & $08 / 26$ & $\begin{array}{l}1 \\
4 \\
3 \\
5 \\
2 \\
1 \\
3 \\
2 \\
1\end{array}$ & $\begin{array}{r}\mathbf{1} \\
5 \\
a \\
13 \\
15 \\
16 \\
19 \\
21 \\
22\end{array}$ & $\begin{array}{l}09 / 01 \\
09 / 06 \\
09 / 07 \\
09 / 08 \\
09 / 13 \\
09 / 14 \\
09 / 20 \\
09 / 22 \\
09 / 29\end{array}$ & $\begin{array}{l}348 \\
407 \\
430 \\
389 \\
373 \\
357 \\
407 \\
389 \\
348\end{array}$ \\
\hline
\end{tabular}


Appendix Table A2.--cont.

\begin{tabular}{|c|c|c|c|c|c|c|c|}
\hline \multirow{2}{*}{ Bran\&/ } & \multirow{2}{*}{$\begin{array}{c}\text { Release } \\
\text { site }\end{array}$} & \multirow{2}{*}{$\begin{array}{l}\text { Number } \\
\text { released }\end{array}$} & \multirow{2}{*}{$\begin{array}{l}\text { Date } \\
\text { released }\end{array}$} & \multicolumn{2}{|c|}{ Recaptures } & \multirow{2}{*}{$\begin{array}{l}\text { Date } \\
\text { recapture }\end{array}$} & \multirow{2}{*}{$\begin{array}{r}\text { Recapture } \\
\text { site (Rkm) }\end{array}$} \\
\hline & & & & No. & Cumulative & & \\
\hline $\mathrm{LD} 7 \mathrm{~S} 3$ & RKm 470 & 1,855 & $09 / 02$ & $\begin{array}{l}I \\
1 \\
1 \\
1 \\
I \\
1\end{array}$ & $\begin{array}{l}\text { I } \\
2 \\
3 \\
4 \\
5 \\
6\end{array}$ & $\begin{array}{l}09 / 08 \\
09 / 14 \\
09 / 15 \\
09 / 21 \\
09 / 22 \\
09 / 29\end{array}$ & $\begin{array}{l}389 \\
357 \\
348 \\
430 \\
389 \\
348\end{array}$ \\
\hline L A E 4 & $\begin{array}{l}\text { RKm } 373 \\
\text { (purse }\end{array}$ & ine) ${ }^{363}$ & $07 / 06$ & $\begin{array}{l}2 \\
a\end{array}$ & $\begin{array}{r}2 \\
10\end{array}$ & $\begin{array}{l}07 / 14 \\
07 / 21\end{array}$ & $\begin{array}{l}389 \\
348\end{array}$ \\
\hline LA E 3 & RKm 348 & 534 & $07 / 07$ & $\begin{array}{l}1 \\
1 \\
1 \\
1\end{array}$ & $\begin{array}{l}1 \\
2 \\
3 \\
4\end{array}$ & $\begin{array}{l}07 / 27 \\
07 / 28 \\
\text { oaf02 } \\
\text { oaf } 17\end{array}$ & $\begin{array}{l}430 \\
389 \\
373 \\
357\end{array}$ \\
\hline L A E 2 & RKm 407 & 1,108 & $07 / 12$ & 1 & 1 & $08 / 24$ & 430 \\
\hline LA E 1 & RKm 430 & 1,655 & $07 / 13$ & $\begin{array}{l}1 \\
1 \\
1 \\
1\end{array}$ & $\begin{array}{l}1 \\
2 \\
3 \\
4\end{array}$ & $\begin{array}{l}07 / 20 \\
07 / 21 \\
07 / 26 \\
08 / 11\end{array}$ & $\begin{array}{l}357 \\
348 \\
407 \\
389\end{array}$ \\
\hline LD E 1 & RKm 389 & 1,786 & $07 / 14$ & $\begin{array}{l}1 \\
1 \\
1 \\
2 \\
1\end{array}$ & $\begin{array}{l}1 \\
2 \\
3 \\
5 \\
6\end{array}$ & $\begin{array}{l}07 / 20 \\
07 / 21 \\
08 / 10 \\
08 / 11 \\
09 / 06\end{array}$ & $\begin{array}{l}357 \\
348 \\
430 \\
389 \\
407\end{array}$ \\
\hline LU E 2 & RKm 357 & 979 & $07 / 20$ & $\begin{array}{l}\mathrm{I} \\
1\end{array}$ & $\begin{array}{l}1 \\
2\end{array}$ & $\begin{array}{l}07 / 26 \\
08 / 03\end{array}$ & $\begin{array}{l}407 \\
357\end{array}$ \\
\hline LD E 3 & $\mathrm{RKm} 348$ & 1,902 & $07 / 21$ & $\begin{array}{l}2 \\
I \\
1 \\
3 \\
1 \\
1 \\
2 \\
2 \\
1 \\
1 \\
1\end{array}$ & $\begin{array}{r}2 \\
3 \\
4 \\
7 \\
a \\
9 \\
11 \\
13 \\
14 \\
15 \\
16\end{array}$ & $\begin{array}{l}07 / 26 \\
07 / 28 \\
0 a f 02 \\
08 / 03 \\
08 / 04 \\
08 / 11 \\
08 / 16 \\
08 / 24 \\
09 / 13 \\
09 / 15 \\
08 / 21\end{array}$ & $\begin{array}{l}407 \\
389 \\
373 \\
357 \\
348 \\
389 \\
373 \\
430 \\
373 \\
348 \\
430\end{array}$ \\
\hline
\end{tabular}


Appendix Table A2.--cont.

\begin{tabular}{|c|c|c|c|c|c|c|c|}
\hline \multirow[b]{2}{*}{ Branda/ } & \multirow{2}{*}{$\begin{array}{l}\text { Release } \\
\text { site }\end{array}$} & \multirow{2}{*}{$\begin{array}{l}\text { Number } \\
\text { released }\end{array}$} & \multirow{2}{*}{$\begin{array}{l}\text { Date } \\
\text { released }\end{array}$} & \multicolumn{2}{|c|}{ Recaptures } & \multirow{2}{*}{$\begin{array}{l}\text { Date } \\
\text { recapture }\end{array}$} & \multirow{2}{*}{$\begin{array}{r}\text { Recapture } \\
\text { site (Rkm) }\end{array}$} \\
\hline & & & & No. & Cumulative & & \\
\hline \multirow[t]{5}{*}{ LA HE 1} & $\mathrm{RKm} 407$ & 735 & $07 / 26$ & 1 & 1 & $07 / 28$ & 389 \\
\hline & & & & 1 & 2 & $08 / 02$ & 373 \\
\hline & & & & 1 & 3 & $08 / 03$ & 357 \\
\hline & & & & 1 & 4 & $08 / 04$ & 348 \\
\hline & & & & 1 & 5 & $08 / 24$ & 430 \\
\hline \multirow{5}{*}{ LA HE 2} & RKm 430 & 289 & $07 / 27$ & 1 & 1 & $08 / 04$ & 348 \\
\hline & & & & 1 & 2 & $08 / 10$ & 430 \\
\hline & & & & 1 & 3 & $08 / 23$ & 407 \\
\hline & & & & 2 & 5 & $08 / 24$ & 430 \\
\hline & & & & 1 & 6 & $09 / 29$ & 348 \\
\hline \multirow[t]{13}{*}{ LA HE 3} & RKm 389 & 1,751 & $07 / 28$ & 1 & 1 & $08 / 02$ & 373 \\
\hline & & & & 2 & 3 & $08 / 03$ & 357 \\
\hline & & & & 3 & 6 & $08 / 04$ & 348 \\
\hline & & & & 1 & 7 & $08 / 11$ & 389 \\
\hline & & & & 1 & $a$ & $08 / 16$ & 373 \\
\hline & & & & 2 & 10 & $08 / 17$ & 357 \\
\hline & & & & 1 & 11 & $08 / 18$ & 348 \\
\hline & & & & I & 12 & $08 / 24$ & 430 \\
\hline & & & & 1 & 13 & $09 / 01$ & 348 \\
\hline & & & & 1 & 14 & 09/06 & 407 \\
\hline & & & & 1 & 15 & 09/07 & 430 \\
\hline & & & & 1 & 16 & $09 / 13$ & 373 \\
\hline & & & & 1 & 17 & $09 / 22$ & 389 \\
\hline \multirow[t]{4}{*}{ LA HE 4} & RKm 373 & 1,194 & $08 / 02$ & 1 & 1 & $08 / 16$ & 373 \\
\hline & & & & 1 & 2 & $08 / 17$ & 357 \\
\hline & & & & 1 & 3 & $09 / 01$ & 348 \\
\hline & & & & 1 & 4 & $09 / 14$ & 357 \\
\hline \multirow[t]{5}{*}{ LD $\mathrm{HE} 1$} & RKm 357 & 604 & $08 / 03$ & 1 & 1 & $08 / 04$ & 348 \\
\hline & & & & 2 & 3 & $08 / 16$ & 373 \\
\hline & & & & 2 & 5 & $08 / 17$ & 357 \\
\hline & & & & 1 & 6 & $09 / 20$ & 407 \\
\hline & & & & 1 & 7 & $09 / 28$ & 357 \\
\hline \multirow[t]{6}{*}{ LD HE 2} & RKm 348 & 931 & $08 / 04$ & 1 & 1 & $08 / 11$ & 389 \\
\hline & & & & & 3 & $08 / 16$ & 373 \\
\hline & & & & 2 & 5 & $08 / 18$ & 348 \\
\hline & & & & 1 & 6 & $09 / 06$ & 407 \\
\hline & & & & 1 & 7 & $09 / 14$ & 357 \\
\hline & & & & 1 & 8 & $09 / 2 l$ & 430 \\
\hline
\end{tabular}


Appendix Table Az.--cont.

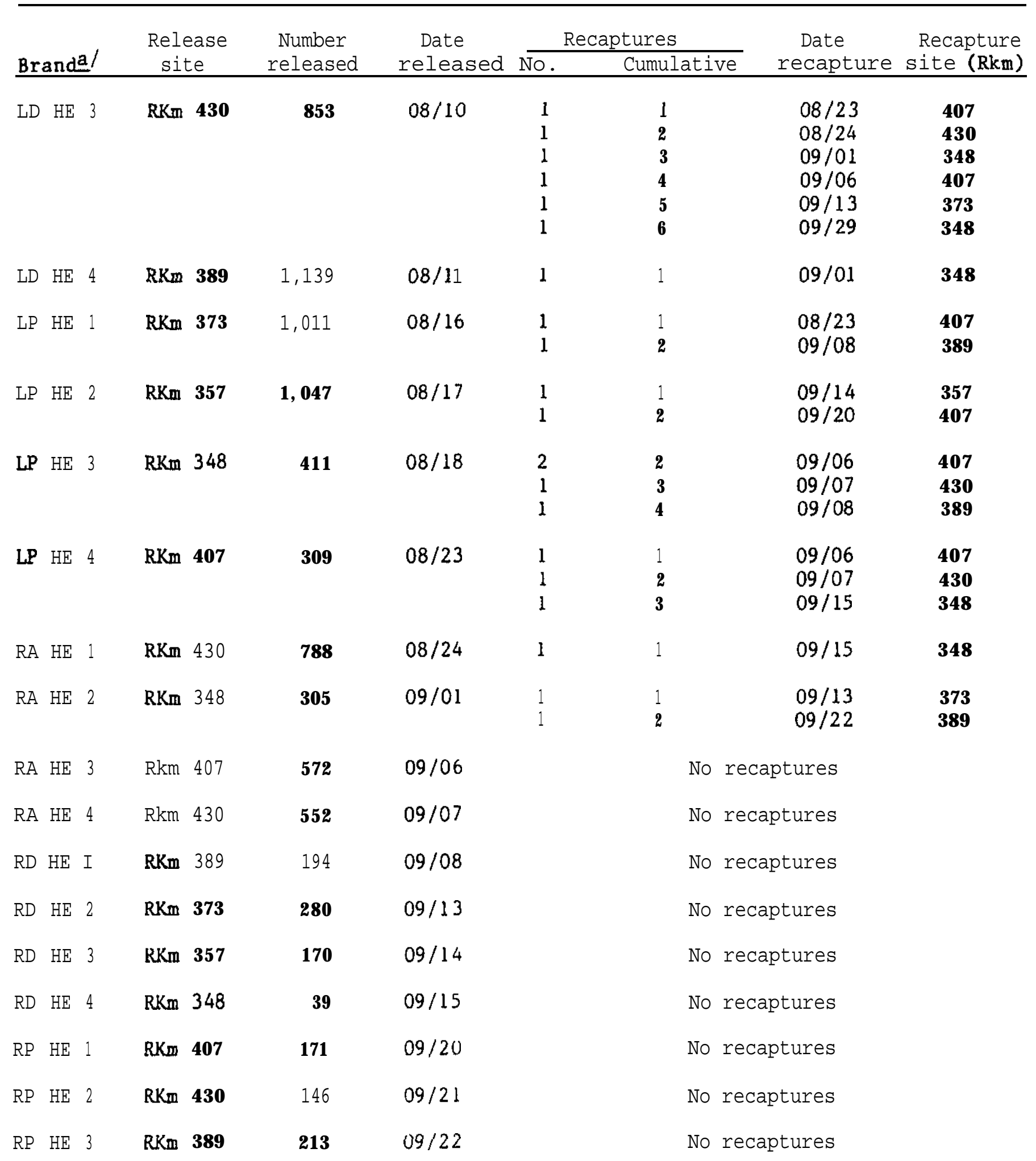


Appendix Table AZ.--cont.

\begin{tabular}{|c|c|c|c|c|c|c|c|}
\hline \multirow[b]{2}{*}{ Branda/ } & \multirow{2}{*}{$\begin{array}{l}\text { Release } \\
\text { site }\end{array}$} & \multirow{2}{*}{$\begin{array}{l}\text { Number } \\
\text { released }\end{array}$} & \multirow{2}{*}{$\begin{array}{l}\text { Date } \\
\text { released }\end{array}$} & \multicolumn{2}{|c|}{ Recaptures } & \multirow{2}{*}{$\begin{array}{c}\text { Date } \\
\text { recapture }\end{array}$} & \multirow{2}{*}{$\begin{array}{r}\text { Recapture } \\
\text { site (Rkm) }\end{array}$} \\
\hline & & & & $\overline{\mathrm{No}}$ & Cumulative & & \\
\hline $\mathrm{RP} \quad \mathrm{HE} \quad 4$ & RKm 357 & 62 & $09 / 28$ & & No $r$ & aptures & \\
\hline IA D 1 & RKm 346 & 103 & $09 / 29$ & & No $r$ & aptures & \\
\hline RA 31 & & & & 1 & 1 & $07 / 27$ & 430 \\
\hline $\mathrm{RA} 33$ & & & & 1 & 1 & $08 / 24$ & 430 \\
\hline
\end{tabular}


Appendix Table A3.--Average daily discharge McNary Dam, 1983.

\begin{tabular}{|c|c|c|c|c|c|c|c|c|c|c|}
\hline Date & $\begin{array}{l}\text { Discharge } \\
\text { (Kcfs) }\end{array}$ & Date & & $\begin{array}{l}\text { Discharge } \\
\text { (Kcfs) }\end{array}$ & Date & & $\begin{array}{l}\text { Discharge } \\
\text { (Kcfs) }\end{array}$ & Date & & $\begin{array}{l}\text { Discharge } \\
\text { (Kcfs) }\end{array}$ \\
\hline $\begin{array}{r}1 \\
\text { June } \\
3 \\
4 \\
5 \\
6 \\
7 \\
a \\
9 \\
10 \\
11 \\
12 \\
13 \\
14 \\
15 \\
16 \\
17 \\
18 \\
19 \\
20 \\
21 \\
22 \\
23 \\
24 \\
25 \\
26 \\
27 \\
28 \\
29 \\
30\end{array}$ & $\begin{array}{l}398.7 \\
367.0 \\
379.3 \\
3 b 8.4 \\
352.9 \\
344.8 \\
323.0 \\
359.8 \\
342.2 \\
343.5 \\
323.6 \\
306.6 \\
306.1 \\
312.3 \\
258.9 \\
268.2 \\
265.4 \\
263.8 \\
245.1 \\
254.9 \\
234.3 \\
240.7 \\
230.4 \\
218.9 \\
206.7 \\
216.5 \\
200.0 \\
210.6 \\
212.4 \\
215.2\end{array}$ & . & $\begin{array}{r}\text { I } \\
2 \\
3 \\
4 \\
5 \\
6 \\
7 \\
8 \\
9 \\
10 \\
11 \\
12 \\
13 \\
14 \\
15 \\
16 \\
17 \\
18 \\
19 \\
20 \\
21 \\
22 \\
23 \\
24 \\
25 \\
26 \\
27 \\
28 \\
29 \\
30 \\
31\end{array}$ & $\begin{array}{l}203.2 \\
178.3 \\
177.9 \\
187.7 \\
207.8 \\
216.0 \\
234.3 \\
213.8 \\
190.3 \\
152.3 \\
182.5 \\
206.9 \\
223.2 \\
204.5 \\
189.2 \\
207.9 \\
211.4 \\
226.9 \\
221.6 \\
230.1 \\
228.7 \\
221.8 \\
203.0 \\
169.4 \\
193.4 \\
213.5 \\
229.6 \\
213.8 \\
218.6 \\
193.8 \\
218.1\end{array}$ & August & $\begin{array}{r}1 \\
2 \\
3 \\
4 \\
5 \\
6 \\
7 \\
8 \\
9 \\
10 \\
11 \\
12 \\
13 \\
14 \\
15 \\
16 \\
17 \\
18 \\
19 \\
20 \\
21 \\
22 \\
23 \\
24 \\
25 \\
26 \\
27 \\
28 \\
29 \\
30 \\
31\end{array}$ & $\begin{array}{r}198.5 \\
196.6 \\
180.4 \\
188.7 \\
180.9 \\
187.5 \\
164.7 \\
181.0 \\
186.8 \\
176.3 \\
179.6 \\
166.9 \\
143.7 \\
145.1 \\
156.9 \\
157.3 \\
178.9 \\
179.1 \\
191.8 \\
186.0 \\
146.5 \\
162.4 \\
137.3 \\
140.8 \\
138.0 \\
128.4 \\
122.7 \\
94.1 \\
105.6 \\
129.1 \\
148.2\end{array}$ & $\begin{array}{l} \\
\\
7 \\
7 \\
7 \\
2 \\
2 \\
2 \\
2 \\
2 \\
2 \\
2 \\
2 \\
2 \\
2 \\
2 \\
2\end{array}$ & $\begin{array}{r}1 \\
2 \\
3 \\
4 \\
5 \\
6 \\
7 \\
8 \\
9 \\
10 \\
11 \\
12 \\
13 \\
14 \\
15 \\
16 \\
17 \\
18 \\
19 \\
20 \\
21 \\
22 \\
23 \\
24 \\
25 \\
26 \\
27 \\
28 \\
29 \\
30\end{array}$ & $\begin{array}{r}171.4 \\
150.4 \\
129.1 \\
94.4 \\
86.8 \\
96.0 \\
140.8 \\
130.5 \\
130.1 \\
151.9 \\
89.3 \\
86.2 \\
120.7 \\
127.5 \\
116.4 \\
116.5 \\
120.1 \\
96.7 \\
121.0 \\
105.2 \\
142.1 \\
111.1 \\
144.4 \\
91.8 \\
69.1 \\
115.0 \\
120.1 \\
114.0 \\
117.8 \\
109.8\end{array}$ \\
\hline
\end{tabular}


APPENDIX B

SUMMARY OF EXPENDITURES - PY 1983 


\section{Category}

Personnel

Travel

Vehicles

Rent, Communications, SLUC, and Utilities

Printing

Contract Services

Supplies and Materials

Support

TOTAL $\underline{\text { Amount }}$

\section{$\$ 95,593.26$}

$2,464.64$

$6,843.46$

$2,620.31$

234.48

$5,269.19$

$25,828.89$

$32,978.66$

$171,832.89$

*Major individual expenditures exceeding $\$ 500.00$.

Item

Coded wire tags
Amount

\$ $2,683.40$ 


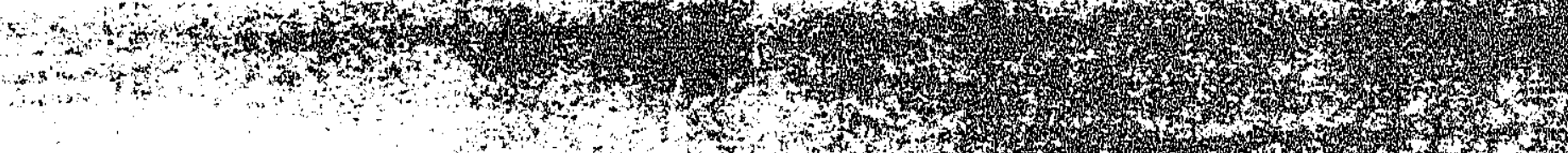

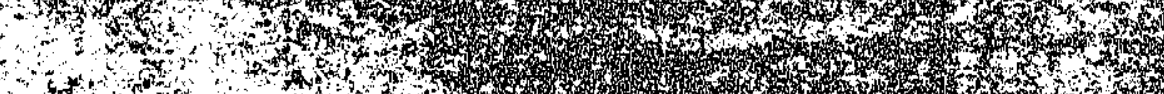
and

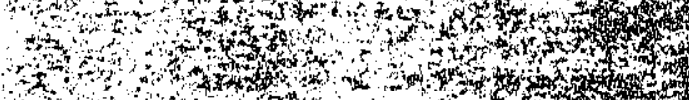
$3 x+2$

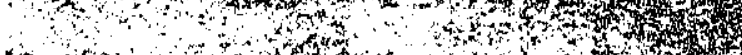

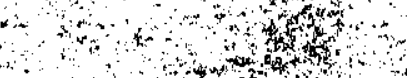

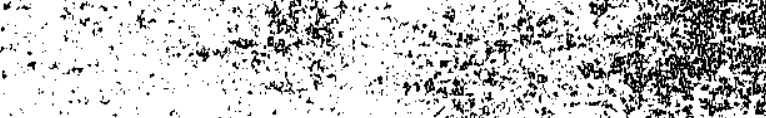

a swan

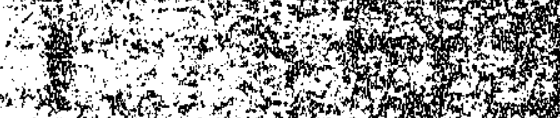

is

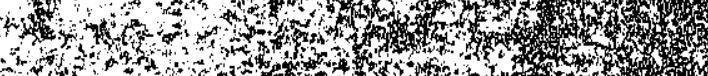

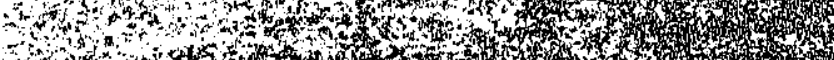

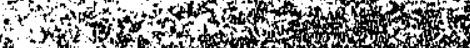

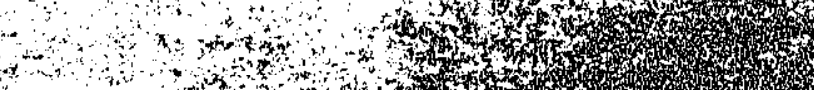

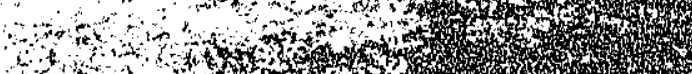

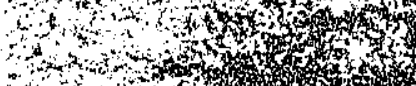

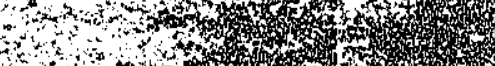

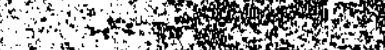

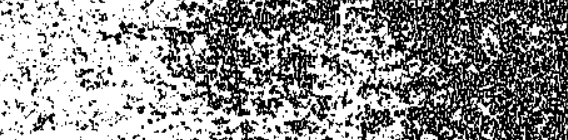

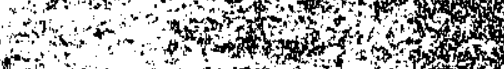

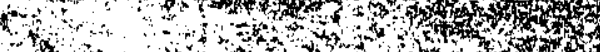

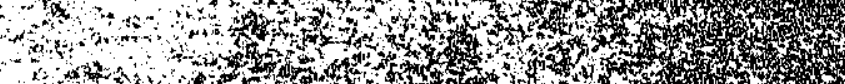

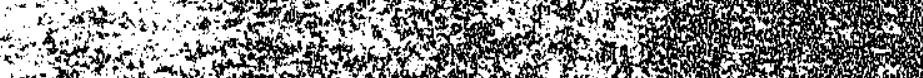

and

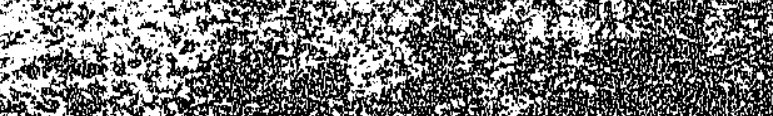

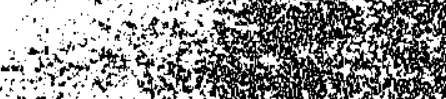

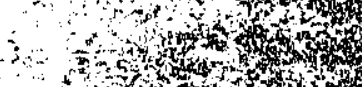

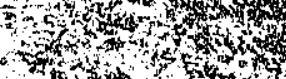

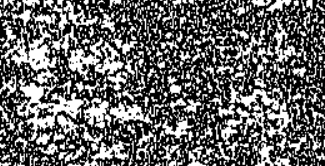

$*$

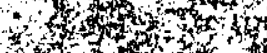
ind

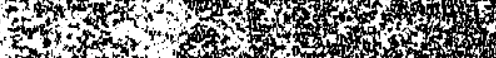

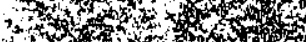

2.

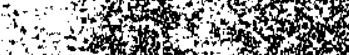

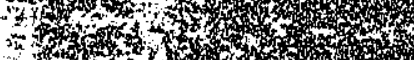

, n

,

ty

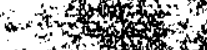

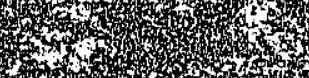

on

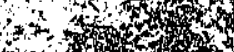

in

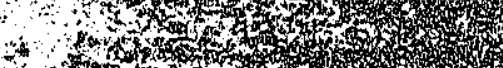

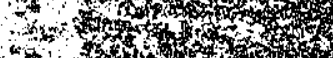

, w

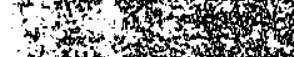

,

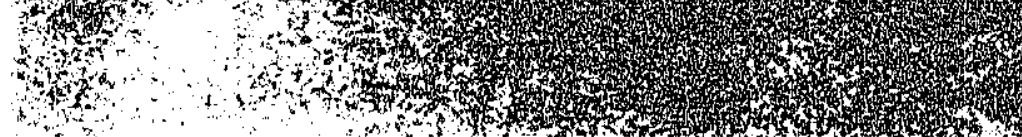
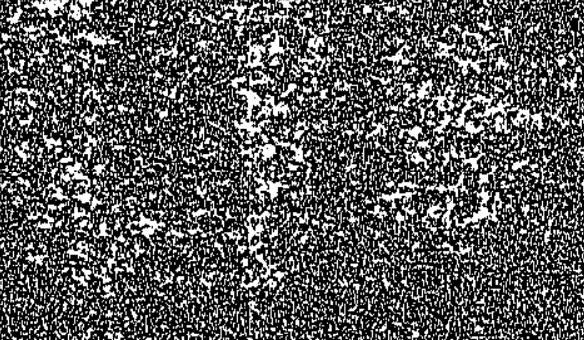

(n) 